\title{
Stokes flow singularity at the junction between impermeable and porous walls
}

\author{
Ludwig C. Nitsche $\dagger$ and Prashanth Parthasarathi \\ Department of Chemical Engineering, The University of Illinois at Chicago, 810 South Clinton Street, \\ Chicago, IL 60607, USA
}

(Received 30 November 2011; revised 25 June 2012; accepted 13 September 2012; first published online 24 October 2012)

For two-dimensional, creeping flow in a half-plane, we consider the singularity that arises at an abrupt transition in permeability from zero to a finite value along the wall, where the pressure is coupled to the seepage flux by Darcy's law. This problem represents the junction between the impermeable wall of the inflow section and the porous membrane further downstream in a spiral-wound desalination module. On a macroscopic, outer length scale the singularity appears like a jump discontinuity in normal velocity, characterized by a non-integrable $1 / r$ divergence of the pressure. This far-field solution is imposed as the boundary condition along a semicircular arc of dimensionless radius 30 (referred to the microscopic, inner length scale). A preliminary numerical solution (using a least-squares variant of the method of fundamental solutions) indicates a continuous normal velocity along the wall coupled with a weaker $1 / \sqrt{r}$ singularity in the pressure. However, inconsistencies in the numerically imposed outer boundary condition indicate a very slow radial decay. We undertake asymptotic analysis to: (i) understand the radial decay behaviour; and (ii) find a more accurate far-field solution to impose as the outer boundary condition. Similarity solutions (involving a stream function that varies like some power of $r$ ) are insufficient to satisfy all boundary conditions along the wall, so we generalize these by introducing linear and quadratic terms in $\log r$. By iterating on the wall boundary conditions (analogous to the method of reflections), the outer asymptotic series is developed through second order. We then use a hybrid computational scheme in which the numerics are iteratively patched to the outer asymptotics, thereby determining two free coefficients in the latter. We also derive an inner asymptotic series and fit its free coefficient to the numerics at $r=0.01$. This enables evaluation of the singular flow field in the limit as $r \rightarrow 0$. Finally, a uniformly valid fit is obtained with analytical formulas. The singular flow field for a solid-porous abutment and the general Stokes flow solutions obtained in the asymptotic analysis are programmed in Fortran for future use as local basis functions in computational schemes. Numerics are required for the intermediate- $r$ regime because the inner and outer asymptotic expansions do not extend far enough toward each other to enable rigorous asymptotic matching. The logarithmic correction terms explain why the leading far-field solution (used in the preliminary numerics) was insufficient even at very large distances.

Key words: boundary integral methods, boundary layer control, porous media 


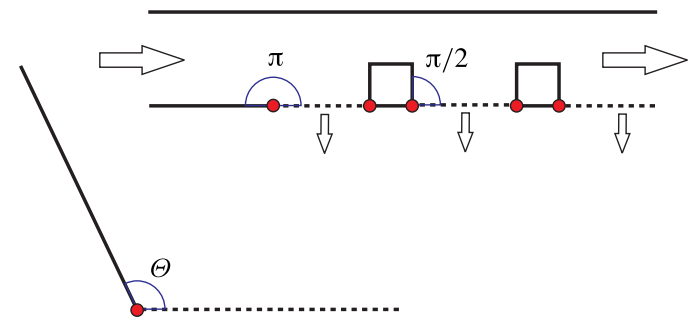

FIgURE 1. (Colour online) Lower left. The general fluid domain is an angular wedge extending indefinitely in the radial coordinate, with the impermeable boundary (solid line) lying along the ray $\theta=\Theta$ and the porous membrane (dotted line) lying along the ray $\theta=0$. Upper right. Schematic diagram of the flow channel in a spiral-wound membrane module. The horizontal arrows indicate tangential flow and the vertical arrows indicate seepage through the porous membrane. The abrupt end of the sealed, entry section of the lower wall corresponds to $\Theta=\pi$ and represents the problem treated in this paper. The case $\Theta=\pi / 2$ would apply to a solid spacer adjoining the membrane, and will be treated in a subsequent paper. The singular points are indicated with large, closed circles.

\section{Introduction}

The flow of fluids in channels and tubes with porous walls or over porous surfaces is central to tangential-flow membrane separations (Yucel \& Turkoglu 1995; Yeh \& Cheng 1999; Miranda \& Campos 2001; Geraldes, Semião \& de Pinho 2002a; Geraldes, Semiao \& de Pinho 2002b; de Pinho, Semião \& Geraldes 2002; Damak et al. 2004a; Pak et al. 2008), boundary-layer control (Taylor 1956; Raghunathan, McIlwain \& Mabey 1991; Raghunathan \& Cooper 2000; Betyaev 2002; Patil \& Ng 2010), blood flow through leaky capillaries in tumors (Pozrikidis 2010) and transpiration cooling. Our interest lies in the fine structure of fluid flow at junctions between impermeable and porous sections of the walls. In the case of spiral-wound membrane modules, such abrupt (effectively discontinuous) transitions in wall permeability occur between the membrane and either: (i) the initial and final impermeable sections of the channel (Miranda \& Campos 2001; Geraldes et al. 2002b); or (ii) transverse filaments of a ladder-type spacer (Geraldes et al. 2002a; Geraldes, Semião \& de Pinho 2002c). As is illustrated in figure 1, these two cases are distinguished by the angle $\left(180^{\circ}\right.$ versus $90^{\circ}$, respectively) at which the impermeable and porous boundaries meet. Both angles arise in a theoretically proposed membrane separation process called antipolarization dialysis (Nitsche \& Zhuge 1995). Our general Stokes flow solutions can be applied to satisfy the relevant boundary conditions for an arbitrary angular wedge, although we focus here on the former case of a flat wall with a discontinuous jump in permeability from zero to a finite value.

Starting with the classic similarity solution of Berman (1953), an extensive theoretical literature has grown around the problem of incompressible, Newtonian liquids flowing in channels or tubes with porous walls. Most of these papers impose the boundary condition of uniform normal flux at the porous wall(s) or direct extensions of this case involving accelerating or normally moving walls (Taylor et al. 1991; Chellam, Wiesner \& Dawson 1992; Yuan \& Finkelstein 1956; Weissberg 1959; Terrill 1964; Gupta \& Levy 1976; Robinson 1976; Skalak \& Wang 1978; Brady 1984; Shih 1987; Zaturska, Drazin \& Banks 1988; Hwang \& Wang 1992; Mellis, Gill \& Belfort 1993; Zaturska \& Banks 1995; Lu 1997; Bujurke, Madalli \& Mulimani 1998; 
Hamza 1999; Ferro \& Gnavi 2000; Majdalani, Zhou \& Dawson 2002; Dauenhauer \& Majdalani 2003; Zheng, Zhao \& Zhang 2007; Asghar, Mushtaq \& Hayat 2010), and do not explicitly treat the porous nature of the walls. Multiple steady solutions are found in certain regimes of the wall Reynolds number, and spatial and temporal stability have been explored.

Some papers treat a more realistic specification of wall permeability, by which the normal flux is proportional to the transmembrane pressure drop (Galowin, Fletcher \& DeSantis 1974; Mellis et al. 1993; Miranda \& Campos 2001; Damak et al. 2004b; King \& Cox 2005; Haldenwang 2007; Pak et al. 2008; Pozrikidis 2010). A finer-scale description replaces the permeable boundary condition imposed on an infinitesimally thin line or surface with an explicit treatment of Darcy or Brinkman flow in the adjacent porous layer of finite thickness (Neale \& Nader 1974; Gupte \& Advani 1997; Deng \& Martinez 2005; Kostoglou \& Karabelas 2008; Chen, Gunzburger \& Wang 2010; Borsi, Farina \& Fasano 2011). At even smaller scales, continuum hydrodynamic models of the microporous structure itself shed light on the appropriate boundary conditions for slip and/or drift velocity at a porous surface (Larson \& Higdon 1986, 1987; Sahraoui \& Kaviany 1992; Pozrikidis 2001, 2005; Liu \& Prosperetti 2011).

Here we shall be satisfied with a permeable boundary condition and consider its consequences for the fine structure of the two-dimensional flow field at the junction with an impermeable wall. The dominance of viscous over inertial forces sufficiently near the junction means that we can rely on the Stokes equations to elucidate the singularity (Hancock, Lewis \& Moffatt 1981). Taylor's paint-scraper discontinuity and a related rotary honing problem, hinged-plate and Jeffery-Hamel flows, eddies and singularities at corners and other classic solutions in creeping flow (Taylor 1960, 1962; Moffatt 1964a; Pan \& Acrivos 1967; Moffatt \& Duffy 1980; Gupta, Manohar \& Noble 1981; Hancock et al. 1981; Harper \& Wake 1983; Hills \& Moffatt 2000) are of the similarity form, which involves a radial power law for the stream function (when expressed in polar coordinates)

$$
\psi(r, \theta)=r^{\lambda} f(\theta) .
$$

Assuming a discontinuous jump from zero to a finite seepage velocity corresponds to $\lambda=1$, and leads to a non-integrable $r^{-1}$ blow-up of the pressure (Nitsche \& Zhuge 1995). This represents an outer asymptotic limit for our problem of a permeable boundary condition. As we shall see below, the leading inner asymptotic solution corresponds to $\lambda=3 / 2$. This leaves the seepage velocity continuous and softens the pressure singularity to $r^{-1 / 2}$. Unfortunately, the similarity form breaks down for even the first correction in both the inner and outer asymptotic solutions. The implementation of usable outer boundary conditions in a hybrid numerical-asymptotic scheme will be seen to require two corrections in the outer field. In the quest for a more general functional form for the stream function, we note the description of logarithmic asymptotic behaviour by Moffatt \& Duffy (1980) in resolving the breakdown of similarity solutions at a special angle for the hinged-plate and Jeffery-Hamel problems. We are also guided by the occurrence of $\log r$ terms in some biharmonic problems from the theory of linear elasticity (Dempsey \& Sinclair 1979, 1981; Dempsey 1995; Stampouloglou \& Theotokoglou 2010; Li \& Wang 2010) and these have started to filter into Stokes flow problems involving corner (Sinclair, Chi \& Shih 2009) and contact-line (Sinclair 2010) singularities. Here we shall go one step further by using quadratic polynomials in $\log r$ to carry the inner and outer 
asymptotic solutions to second order. (The calculations for a longer power series in $\log r$ would be analogous but increasingly tedious.)

Whether for planar or tubular geometries, mathematical models of flow and/or mass transfer in nanofiltration and reverse osmosis typically impose an inflowing velocity profile (uniform or parabolic) at the cross section that marks the start of the membrane (Sherwood et al. 1965; Gupta \& Levy 1976; de Pinho et al. 2002; Geraldes et al. 2002b; Damak et al. 2004a,b; Kim 2007; Pak et al. 2008), and do not treat the impermeable section of the wall that lies upstream of this boundary condition. In those papers that explicitly model the flow rearrangement between impermeable and porous sections of the wall (Miranda \& Campos 2001) or impermeable spacers adjoining the porous membrane at right angles (Geraldes et al. 2002a,c), the singularity mentioned above is not resolved in detail by the numerical scheme. We expect that coupling between the pressure singularities and osmotic effects would affect the solute concentration field. This is important from a practical perspective because the upstream portion of the membrane (where concentration-polarization layers are thinnest) is the most productive, and affects the transport process farther downstream.

Computational methods in fluid mechanics are increasingly relying on local asymptotic expansions to resolve fine-scale features such as singularities at corners. The stream function is typically expanded in a power series of similarity forms (1.1) and the coefficients determined by iterative and other procedures for matching with the numerical solution (Hawa \& Rusak 2002; Shi, Breuer \& Durst 2004; Shapeev \& Lin 2009). Our specific solution for the impermeable-porous junction (and our more general solutions involving the first and second powers $\log r$ ) would be useful in resolving fine structures without the need for local mesh refinement.

\subsection{Overview of the paper}

In the remainder of this introduction the Stokes flow problem is formulated mathematically $(\S 1.2)$. In $\S 2$ we present a first, purely numerical solution and indicate why further asymptotic work is desirable to: (i) formulate workable outer boundary conditions; and (ii) resolve the weakly singular inner behaviour as $r \rightarrow 0$. Preparatory to both outer and inner asymptotic expansions, mathematical preliminaries in $\S 3$ generalize the well-known similarity solution of the two-dimensional Stokes equations (Taylor 1960, 1962; Moffatt 1964a; Pan \& Acrivos 1967; Moffatt \& Duffy 1980; Gupta et al. 1981; Hancock et al. 1981; Harper \& Wake 1983) by introducing a series in powers of $\log r$, carried through quadratic terms. In $\S 4$ we derive a regular perturbation series for the outer solution through second order by iterating on the seepage condition, in a manner that is reminiscent of the method of reflections (Happel \& Brenner 1991; Kim \& Karrila 2005). This procedure yields three basis functions that are consistent with all of the boundary conditions. Outer information is sufficient to specify the coefficient of only the first of these. Thus, one must defer determination of the remaining two coefficients to patching with the numerics, which is done with an iteration scheme in $\S 5$. Analytical results for the inner solution are considered in $\S 6$, and a uniformly valid set of analytical formulas is developed in $\S 7$. Concluding remarks are offered in $\S 8$.

\subsection{Statement of the problem}

For generality, suppose that the fluid domain comprises an arbitrary angular wedge, with the impermeable boundary lying along the ray $\theta=\Theta$ and the porous membrane lying along the ray $\theta=0$ (figure 1). In our problem $\Theta=\pi$, representing the abrupt end of the sealed, entry section of the membrane in a spiral-wound desalination 
module. (The case $\Theta=\pi / 2$ would apply to a solid spacer adjoining the membrane, and will be treated in a subsequent paper.) The porous membrane is characterized by permeability $\kappa$ and thickness $\ell$, in terms of which seepage is described by Darcy's law.

$$
V_{n}=\frac{\kappa}{\mu \ell} \Delta P .
$$

Based upon the following characteristic scales,

Characteristic length: $\kappa / \ell$

Characteristic pressure: $\mathscr{P}^{*}$

Characteristic velocity: $\mathscr{P}^{*} \kappa /(\mu \ell)$

we pose the following dimensionless Stokes flow problem:

$$
\begin{array}{rlll}
\nabla^{2} \boldsymbol{v}=\nabla p, & 0<r<\infty, & 0<\theta<\Theta & \text { Momentum } \\
\frac{\partial\left(r v_{r}\right)}{\partial r}+\frac{\partial v_{\theta}}{\partial \theta}=0, & 0<r<\infty, & 0<\theta<\Theta & \text { Continuity } \\
v_{r}(r, \Theta)=0, & 0<r<\infty & & \text { No slip } \\
v_{\theta}(r, \Theta)=0, & 0<r<\infty & & \text { Impermeability } \\
v_{r}(r, 0)=0 & 0<r<\infty & & \text { No slip } \\
v_{\theta}(r, 0)=-p(r, 0), & 0<r<\infty & & \text { Seepage } \\
p(r, \theta) \rightarrow p^{\{1\}}(r, \theta), & r \rightarrow \infty, & 0<\theta<\Theta & \text { Far field } \\
\boldsymbol{v}(r, \theta) \rightarrow \boldsymbol{v}^{\{1\}}(r, \theta), & r \rightarrow \infty, & 0<\theta<\Theta & \text { Far field. }
\end{array}
$$

For consistency with subsequent developments, the far-field conditions (1.12) and (1.13) invoke notation that has not yet been defined (see $\S 4$ ). This outer asymptotic limit is the well-known similarity solution for a discontinuous jump from zero to unit seepage velocity along the wall (Taylor 1960, 1962; Moffatt 1964a; Pan \& Acrivos 1967; Hancock et al. 1981; Gupta et al. 1981; Harper \& Wake 1983; Nitsche \& Zhuge 1995), the latter matching the imposed far-field pressure according to the dimensionless form of Darcy's law (1.11).

$$
\begin{array}{r}
p^{\{1\}}(r, \theta)=1-\left(\frac{2}{\pi}\right) \frac{\cos \theta}{r} \\
v_{r}^{\{1\}}(r, \theta)=\left(\frac{\theta}{\pi}-1\right) \sin \theta \\
v_{\theta}^{\{1\}}(r, \theta)=\left(\frac{\theta}{\pi}-1\right) \cos \theta-\frac{\sin \theta}{\pi} \\
\tau_{\theta r}^{\{1\}}(r, \theta)=\left(\frac{2}{\pi}\right) \frac{\sin \theta}{r} .
\end{array}
$$

In $\S 1$ of the supplementary material (supplementary material available at journals. cambridge.org/flm) we explain why slip at the porous wall (Beavers \& Joseph 1967; Saffman 1971; Jones 1973; Beavers, Sparrow \& Masha 1974; Neale \& Nader 1974; Singh \& Laurence 1979a,b; Nield 1983; Chellam et al. 1992; Chellam, Wiesner \& Dawson 1995; Schmitz \& Prat 1995; Yucel \& Turkoglu 1995; Yeh \& Cheng 1999; Chellam \& Liu 2006; Pak et al. 2008; Nield 2009; Chen et al. 2010; Borsi et al. 2011) should not alter the results obtained with the no-slip condition (1.10). Although 


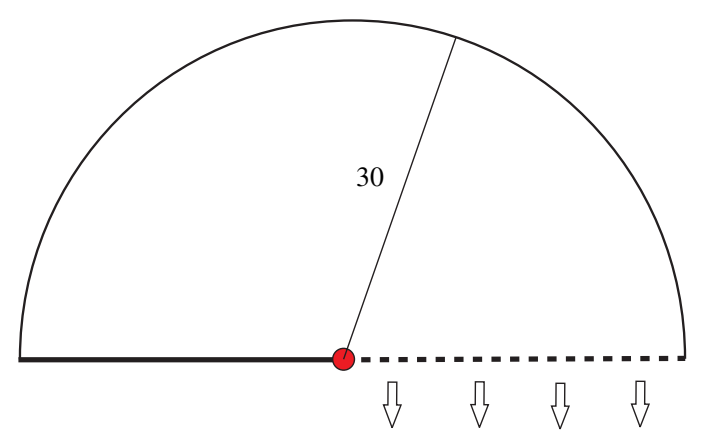

FIgURE 2. (Colour online) Domain and boundary conditions for the numerical scheme. We impose $p$ and $v_{\theta}$ along the semicircular arc. The solid wall (thick, solid line) has no seepage and no slip. The porous wall (thick, dotted line) has seepage but no slip.

the Mellin transform yields solutions for a variety of Stokes flow problems posed in angular wedges (Moffatt 1964b; Moffatt \& Duffy 1980), such an attack cannot be mounted on the problem (1.6)-(1.13). This conclusion is explained in $\S 2$ of the supplementary material.

\section{Initial, purely numerical solution}

For numerical purposes the Stokes boundary-value problem (1.6)-(1.13) is posed on a finite half-disc $0<r<30,0<\theta<\pi$; see figure 2. There are two main issues: (i) solution of the Stokes problem for prescribed boundary conditions; and (ii) formulation of suitable outer boundary conditions along the arc $r=30$.

\subsection{Hybrid fundamental-solution/boundary-integral method}

By the method of fundamental solutions (MFS), linear elliptic boundary-value problems are solved by superposing singular solutions (poles) of the partial differential equation (PDE) that are positioned outside the domain (Kupradze 1964; Kupradze \& Aleksidze 1964; Aleksidze 1966; Kołodziej \& Kleiber 1989). When imposing the boundary conditions at discrete points lying at the edges of the domain, singularities are thereby avoided in the resultant linear equations to be solved for the coefficients of the poles (i.e. singularity strengths). Compared with simple boundary collocation, an overabundance of boundary points leads to a linear least-squares problem, which is generally more robust (Burgess \& Mahajerin 1984; Bogomolny 1985; Nitsche \& Brenner 1990; Nitsche \& Zhuge 1995; Saavedra \& Power 2003, 2004; Alves \& Silvestre 2004; Alves 2009). Pithy discussions of the trade-off between accuracy (favouring singularities farther from the boundary) and conditioning of the matrix (favouring singularities closer to the boundary) are provided by Bogomolny (1985), Saavedra \& Power (2004) and Alves (2009); the last paper also mentions Tikhonov regularization as a means of ameliorating the conditioning issue. When the positions of the poles enter into the error criterion along with their strengths, a nonlinear optimization is required (Mathon \& Johnston 1977; Han \& Olson 1987; Fairweather \& Karageorghis 1998). An adaptive multiple-regression scheme has also been described (Saavedra \& Power 2004).

For interior (exterior) problems, distributing the poles over an externally (internally) displaced auxiliary boundary should yield a sufficient basis (Burgess \& Mahajerin 1984; Kołodziej \& Kleiber 1989; Alves 2009), although star-shaped clusters inside 
spherical particles were used by Da̧broś (1985) to model three-dimensional Stokes flows. (The clusters could be interpreted as yielding finite-difference approximations of certain multipoles (Gluckman, Pfeffer \& Weinbaum 1971; Yan et al. 1987; Dassios \& Hadjinicolaou 2002).) Essentially all MFS treatments of Stokes flow use only point forces (Stokeslets) as the poles. The paper by Da̧broś (1985) and two other papers (Nitsche \& Brenner 1990; Nitsche \& Zhuge 1995) were unusual in augmenting the point force with the point source, which is the fundamental solution for potential flow. The rationale for enlarging the basis was that all velocity fields from potential flow furnish solutions for the Stokes equations when combined with a vanishing (or uniform) pressure field.

Discrete poles correspond to a collection of delta-function spikes for the lineal density of singularity strength along the auxiliary boundary. Our numerical experience with MFS suggests that matrix conditioning and robustness improve with increasing regularity of the lineal density function. Thus, we employ a continuous, piecewiselinear density of both the potential and Stokes singularities over contiguous line segments that form a polygonal auxiliary boundary.

Collect in a matrix-valued point function $\boldsymbol{G}(\boldsymbol{r})$ the pressure and velocity fields for the point source, point force in the $+x$ direction and point force in the $+y$ direction. The first (row) index $i$ refers to the component of the flow field and the second (column) index $j$ indicates the type of singularity, namely

Rows

Columns

$i=0$ : pressure

$$
j=0 \text { : point source }
$$

$i=1: \quad x$ component of velocity $j=1$ : point force in the $+x$ direction

$i=2$ : $\quad y$ component of velocity $j=2$ : point force in the $+y$ direction

$$
\boldsymbol{G}=\left(\begin{array}{lll}
G_{0,0} & G_{0,1} & G_{0,2} \\
G_{1,0} & G_{1,1} & G_{1,2} \\
G_{2,0} & G_{2,1} & G_{2,2}
\end{array}\right)=\left(\begin{array}{lll}
0 & 2 x / r^{2} & 2 y / r^{2} \\
x / r^{2} & x^{2} / r^{2}-\ln r & x y / r^{2} \\
y / r^{2} & x y / r^{2} & y^{2} / r^{2}-\ln r
\end{array}\right)
$$

A three-point basis function $\boldsymbol{F}(\boldsymbol{r} ; \boldsymbol{a}, \boldsymbol{b}, \boldsymbol{c})$ is obtained by linearly ramping up the lineal density of singularity strength from zero to unity over the first line segment $\overrightarrow{a b}$, and then ramping the density back down to zero over the adjoining, second segment $\overrightarrow{\boldsymbol{b} \boldsymbol{c}}$ :

$$
\begin{aligned}
\boldsymbol{F}(\boldsymbol{r} ; \boldsymbol{a}, \boldsymbol{b}, \boldsymbol{c})= & \|\boldsymbol{b}-\boldsymbol{a}\| \int_{0}^{1} s \boldsymbol{G}[\boldsymbol{r}-\boldsymbol{a}-s(\boldsymbol{b}-\boldsymbol{a})] \mathrm{d} s \\
& +\|\boldsymbol{c}-\boldsymbol{b}\| \int_{0}^{1}(1-s) \boldsymbol{G}[\boldsymbol{r}-\boldsymbol{b}-s(\boldsymbol{c}-\boldsymbol{b})] \mathrm{d} s .
\end{aligned}
$$

The ramp-up (ramp-down) segment of each basis function is also the ramp-down (ramp-up) segment of its immediately adjacent predecessor (successor). When all of them are superposed, the partially overlapping hat-functions of singularity density yield a continuous, piecewise-linear distribution.

The basis function (2.2) can be evaluated in closed form (appendix A), and has the following weakly singular behaviour along the segments $\overrightarrow{\boldsymbol{a} \boldsymbol{b}}$ and $\overrightarrow{\boldsymbol{b} \boldsymbol{c}}$; cf. Pozrikidis (1992). (i) The velocity field is continuous at all points. (ii) The pressure field suffers a finite jump discontinuity in passing normally across each line segment. (iii) When the points $\boldsymbol{a}, \boldsymbol{b}$ and $\boldsymbol{c}$ are colinear, the one-sided limit of the pressure (approaching the line in the normal direction from one side or the other) is continuous when regarded 


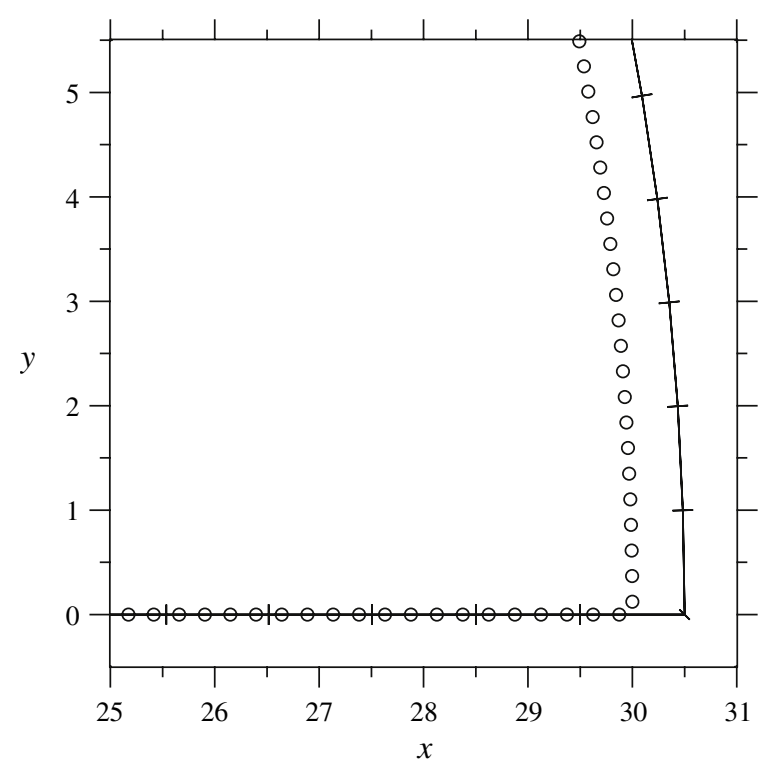

FIGURE 3. Boundary points and singularity segments for the semicircular domain $0<r<$ $30,0<\theta<\pi$; cf. figure 2 . Along the $x$-axis, the boundary points lie on the singularity segments, and the segment length is reduced smoothly from 1.0 near the corners $x= \pm 30$ to 0.001 near the origin. Along the semicircular arc a uniform mesh is used, with the singularity segments lying outboard of the boundary points. In this diagram we zoom in on the corner $(x, y)=(30,0)$.

as a function of tangential position along the line. Otherwise the pressure blows up logarithmically approaching the angular joint $\boldsymbol{b}$.

When local refinement is required to resolve fine structure, which is the case for our problem, schemes that couple the spacing between poles to their distance from the physical boundary can lead to an oddly curved auxiliary boundary; cf. $\S \S 3.6$, 5.2.3 and figure 6 in the paper by Alves (2009). Although our initial trials with such a scheme proved eminently workable, the numerics can be simplified by taking the weakly singular limit wherein the auxiliary boundary moves infinitesimally close to the physical boundary over its straight sections: the porous membrane $(\theta=0)$ and the impermeable wall $(\theta=\pi)$. Colinearity of the boundary segments then guarantees regularity of the pressure profile along the $x$-axis.

Equally spaced points along the arc $r=30$ suffice for the far-field conditions. We keep the corresponding polygonal section of the auxiliary boundary outboard of the boundary points to avoid the aforementioned pressure singularities at the vertices; see figure 3. Thus, our numerical approach is similar to a single-layer boundary-integral equation (BIE) along the $x$-axis, while employing MFS along the semicircular arc. With analytical formulas for the basis function (2.2) in hand, whether the singularity segments lie on or outboard of the physical boundary is irrelevant to implementing the subsequent linear least-squares scheme.

For the finite domain shown in figure 2, the discretization parameters listed in figure 3 gave 376 boundary segments and 1500 boundary points in all. The numerical scheme was validated against the leading outer solution $p^{\{1\}}, v_{r}^{\{1\}}, v_{\theta}^{\{1\}}$ from (1.14)-(1.16); see $\S 3$ of the supplementary material for details and results on this test problem. 


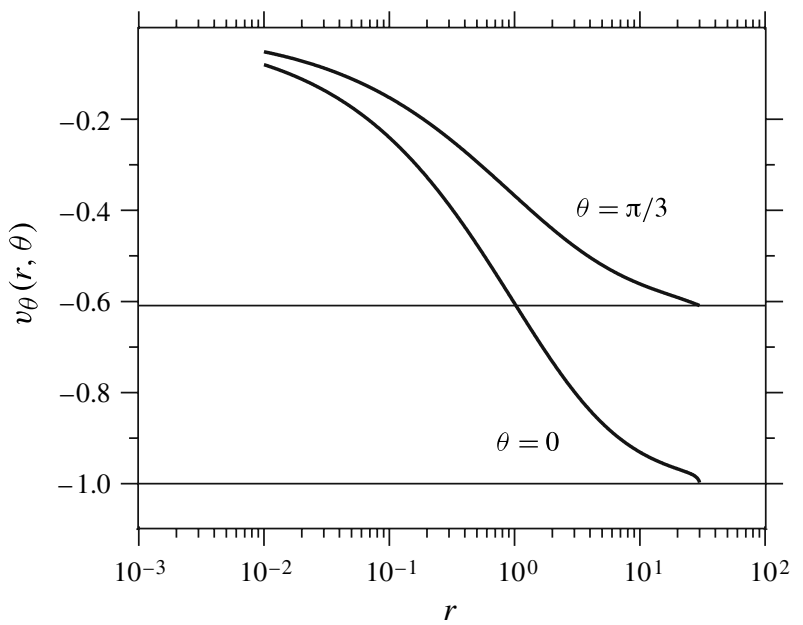

FIGURE 4. Numerically calculated angular velocity component plotted as a function of radius $r$ for $\theta=0, \pi / 3$ (thick lines). Thin lines indicate the leading far-field solution (1.16) that serves as a boundary condition at $r=30$. Note the obviously discontinuous slope where each numerical curve meets the corresponding outer analytical curve.

\subsection{Preliminary numerical solution for a porous wall}

In applying our numerical technique to the wall-seepage problem (1.6)-(1.13), it is initially tempting to replace the far-field, asymptotic-limit conditions (1.12), (1.13) with stipulations at the finite radius of the domain, as worked well for the less challenging test problem described above:

$$
p(30, \theta)=p^{\{1\}}(30, \theta), \quad v_{\theta}(30, \theta)=v_{\theta}^{\{1\}}(30, \theta) \quad(0<\theta<\pi) .
$$

Unfortunately, we now encounter difficulties; see figures 4-6. The numerical curves of $v_{\theta}$ versus $r$ in figure 4 do not level off sufficiently within the radius $r=30$ : they meet their imposed boundary values at appreciable slopes. Indeed, the curve for $\theta=0$ bends down sharply to do so. Since $v_{r}$ was not imposed as a boundary condition at $r=30$, it is not surprising that the numerical curves in figure 5 do not even reach their horizontal asymptotes. Figure 6 shows a breakdown of the outer pressure boundary condition, especially near the porous membrane (i.e. small $\theta$ ). Numerical trials (not shown here) indicate that inconsistency between the calculated $v_{r}$ and the leading outer solution $v_{r}^{\{1\}}$ persist even to $r=300$. Despite these limitations, the numerical solution does indicate the inner asymptotic behaviour: $r^{1 / 2}$ scaling of the pressure profile along the porous membrane and $r^{-1 / 2}$ scaling elsewhere (figure 7); see Parthasarathi (2008). Limitations of the purely numerical approach motivate the outer asymptotic development in $\S 4$.

\section{Mathematical preliminaries}

For the stream-function formulation of the two-dimensional Stokes equations,

$$
\begin{gathered}
\nabla^{4} \psi=\left(\frac{\partial^{2}}{\partial r^{2}}+\frac{1}{r} \frac{\partial}{\partial r}+\frac{1}{r^{2}} \frac{\partial^{2}}{\partial \theta^{2}}\right)^{2} \psi=0 \\
v_{r}=-\frac{1}{r} \frac{\partial \psi}{\partial \theta}, \quad v_{\theta}=\frac{\partial \psi}{\partial r}, \quad \frac{1}{\mu} \frac{\partial p}{\partial r}=-\frac{1}{r} \frac{\partial}{\partial \theta}\left(\nabla^{2} \psi\right)
\end{gathered}
$$




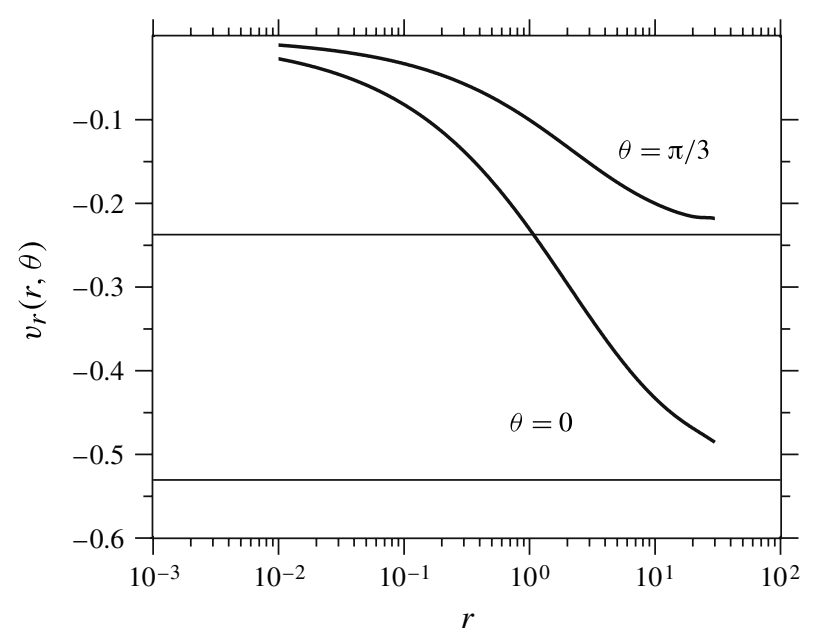

FIGURE 5. Mismatch between the numerically calculated radial velocity component and the corresponding analytical formula (1.15) at $r=30$. The latter is consistent with the pressure and angular velocity component imposed as boundary conditions at $r=30$; see (1.14) and (1.16).

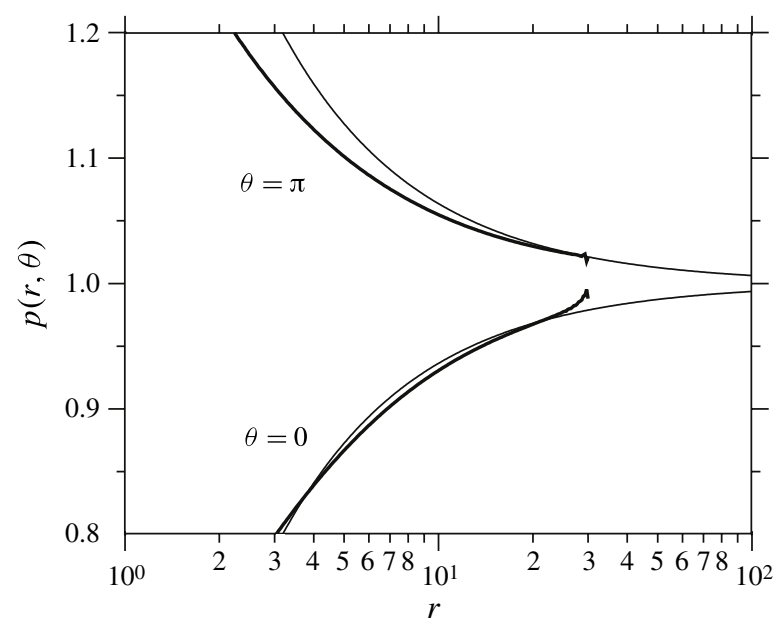

FIGURE 6. Numerically calculated pressure plotted as a function of radius $r$ for $\theta=0, \pi$ (thick lines). Thin lines indicate the leading outer solution (1.14) that provides the pressure boundary condition at $r=30$. Note how the boundary condition breaks down for $\theta=0$.

all solutions considered in this paper can be written in the general form

$$
\psi(r, \theta)=\left\{F(\theta)(\ln r)^{2}+G(\theta) \ln r+H(\theta)\right\} r^{\omega+1} .
$$

The logarithmic terms in $r$ generalize the well-known similarity form (Taylor 1960, 1962; Moffatt 1964a; Pan \& Acrivos 1967; Moffatt \& Duffy 1980; Gupta et al. 1981; Hancock et al. 1981; Harper \& Wake 1983; Shi et al. 2004; Shapeev \& Lin 2009) and are required for iterative satisfaction of the seepage condition (1.11) along the porous membrane. In resolving the breakdown of similarity solutions at a special 


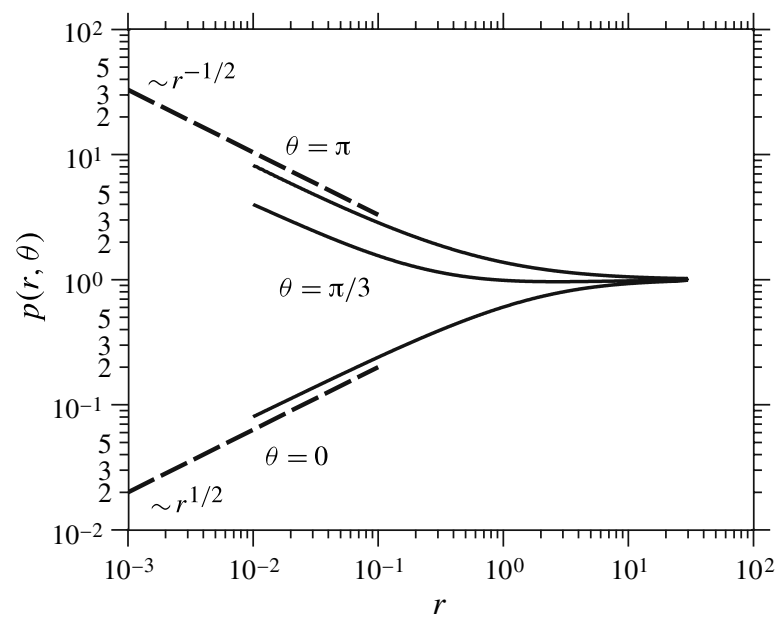

FIGURE 7. Numerically calculated pressure plotted as a function of radius $r$ for $\theta=$ $0, \pi / 3, \pi$. These curves strongly suggest an $r^{1 / 2}$ inner scaling of the pressure at $\theta=0$ and $r^{-1 / 2}$ scaling at other angles.

angle for the hinged-plate and Jeffery-Hamel problems, Moffatt \& Duffy (1980) uncovered logarithmic asymptotic behaviour. The first power of $\ln r$ also appears in some biharmonic problems from the theory of linear elasticity (Dempsey \& Sinclair 1979, 1981; Dempsey 1995; Stampouloglou \& Theotokoglou 2010; Li \& Wang 2010) and these have started to filter into Stokes flow problems involving corner (Sinclair et al. 2009) and contact-line (Sinclair 2010) singularities. We will need the second power of $\ln r$ as well. To compact the subsequent expressions, we introduce three differential operators:

$$
\begin{gathered}
\mathscr{L}_{1}=\frac{\mathrm{d}^{4}}{\mathrm{~d} \theta^{4}}+2\left(1+\omega^{2}\right) \frac{\mathrm{d}^{2}}{\mathrm{~d} \theta^{2}}+\left(1-\omega^{2}\right)^{2} \\
\mathscr{L}_{2}=\frac{\mathrm{d}^{3}}{\mathrm{~d} \theta^{3}}+(1+\omega)^{2} \frac{\mathrm{d}}{\mathrm{d} \theta} \\
\mathscr{L}_{3}=\frac{\mathrm{d}^{2}}{\mathrm{~d} \theta^{2}}+\left(1-\omega^{2}\right) .
\end{gathered}
$$

The PDE (3.1) leads to three ordinary differential equations (ODEs) for the angular functions:

$$
\begin{gathered}
\mathscr{L}_{1}[F(\theta)]=0 \\
\mathscr{L}_{1}[G(\theta)]=-8 \omega F^{\prime \prime}(\theta)-8 \omega\left(\omega^{2}-1\right) F(\theta) \\
\mathscr{L}_{1}[H(\theta)]=-4 F^{\prime \prime}(\theta)-\left(12 \omega^{2}-4\right) F(\theta)-4 \omega G^{\prime \prime}(\theta)-4 \omega\left(\omega^{2}-1\right) G(\theta) .
\end{gathered}
$$

In terms of the angular functions, the velocity, pressure and shear stress are given by

$$
\begin{gathered}
v_{r}(r, \theta)=-r^{\omega}\left\{F^{\prime}(\theta)(\ln r)^{2}+G^{\prime}(\theta) \ln r+H^{\prime}(\theta)\right\} \\
v_{\theta}(r, \theta)=r^{\omega}\left\{[(1+\omega) F(\theta)](\ln r)^{2}+[(1+\omega) G(\theta)+2 F(\theta)] \ln r\right. \\
+[(1+\omega) H(\theta)+G(\theta)]\}
\end{gathered}
$$




$$
\begin{aligned}
p(r, \theta)= & \frac{r^{\omega-1}}{1-\omega}\left\{\left[\mathscr{L}_{2}[F(\theta)]\right](\ln r)^{2}+\left[\mathscr{L}_{2}[G(\theta)]+\frac{2 \mathscr{L}_{2}[F(\theta)]}{1-\omega}+4(1+\omega) F^{\prime}(\theta)\right] \ln r\right. \\
& +\left[\mathscr{L}_{2}[H(\theta)]+\frac{\mathscr{L}_{2}[G(\theta)]}{1-\omega}+\frac{2 \mathscr{L}_{2}[F(\theta)]}{(1-\omega)^{2}}\right. \\
& \left.\left.+2(1+\omega) G^{\prime}(\theta)+2\left(\frac{3+\omega}{1-\omega}\right) F^{\prime}(\theta)\right]\right\} \\
& \tau_{\theta r}(r, \theta)=-r^{\omega-1}\left\{\left[\mathscr{L}_{3}[F(\theta)]\right](\ln r)^{2}+\left[\mathscr{L}_{3}[G(\theta)]-4 \omega F(\theta)\right] \ln r\right. \\
& \left.+\left[\mathscr{L}_{3}[H(\theta)]-2 \omega G(\theta)-2 F(\theta)\right]\right\} .
\end{aligned}
$$

If we define arrays for free constants

$$
\boldsymbol{a}=\left(\begin{array}{l}
a_{1} \\
a_{2} \\
a_{3} \\
a_{4}
\end{array}\right), \quad \boldsymbol{b}=\left(\begin{array}{l}
b_{1} \\
b_{2} \\
b_{3} \\
b_{4}
\end{array}\right), \quad \boldsymbol{c}=\left(\begin{array}{l}
c_{1} \\
c_{2} \\
c_{3} \\
c_{4}
\end{array}\right)
$$

and basis functions

$$
\begin{aligned}
& \boldsymbol{\kappa}_{n}(\theta ; \omega)=\left(\begin{array}{c}
(\omega+1)^{n} \cos [(\omega+1) \theta] \\
(\omega+1)^{n} \sin [(\omega+1) \theta] \\
(\omega-1)^{n} \cos [(\omega-1) \theta] \\
(\omega-1)^{n} \sin [(\omega-1) \theta]
\end{array}\right), \\
& \lambda_{n}(\theta ; \omega)=\left(\begin{array}{c}
-(\omega+1)^{n} \sin [(\omega+1) \theta] \\
(\omega+1)^{n} \cos [(\omega+1) \theta] \\
-(\omega-1)^{n} \sin [(\omega-1) \theta] \\
(\omega-1)^{n} \cos [(\omega-1) \theta]
\end{array}\right),
\end{aligned}
$$

then the general solutions of the ODEs (3.7)-(3.9) can be written in compact form:

$$
\begin{gathered}
F(\theta ; \omega, \boldsymbol{a})=\boldsymbol{a} \cdot\left[\boldsymbol{\kappa}_{0}(\theta ; \omega)\right] \\
G(\theta ; \omega, \boldsymbol{a}, \boldsymbol{b})=\boldsymbol{a} \cdot\left[2 \theta \boldsymbol{\lambda}_{0}(\theta ; \omega)\right]+\boldsymbol{b} \cdot\left[\boldsymbol{\kappa}_{0}(\theta ; \omega)\right] \\
H(\theta ; \omega, \boldsymbol{a}, \boldsymbol{b}, \boldsymbol{c})=\boldsymbol{a} \cdot\left[-\theta^{2} \boldsymbol{\kappa}_{0}(\theta ; \omega)\right]+\boldsymbol{b} \cdot\left[\theta \boldsymbol{\lambda}_{0}(\theta ; \omega)\right]+\boldsymbol{c} \cdot\left[\boldsymbol{\kappa}_{0}(\theta ; \omega)\right] .
\end{gathered}
$$

By substituting these solutions into (3.10)-(3.13), we obtain lengthy, explicit formulas for $v_{r}(r, \theta), v_{\theta}(r, \theta), p(r, \theta)$ and $\tau_{r \theta}(r, \theta)$ in terms of the free constants $\boldsymbol{a}, \boldsymbol{b}$ and $\boldsymbol{c}$. These formulas appear in appendix B. For specific boundary conditions along the impermeable wall $(\theta=\pi)$ and the permeable membrane $(\theta=0)$, solution of the relevant simultaneous, linear algebraic equations yields $\boldsymbol{a}, \boldsymbol{b}, \boldsymbol{c}$. Special cases of the boundary conditions lead to the flow field 'building blocks' $\left\{\boldsymbol{v}^{\{i\}}(\boldsymbol{r}), p^{\{i\}}(\boldsymbol{r})\right\}$ listed in $\S 4.1$, which are then used to construct basis functions $\left\{\boldsymbol{v}^{[k]}(\boldsymbol{r}), p^{[k]}(\boldsymbol{r})\right\}$ for the outer solution in $\S 4.2$.

\section{Outer asymptotic solution}

The outer solution for the numerics is superposed from three basis functions $\left\{\boldsymbol{v}^{[k]}, p^{[k]}\right\}(k=0,1,2)$, each of which is obtained as a regular perturbation series. We introduce a notational hierarchy in which the three levels are distinguished by the type of brackets (i.e. square, round or curly) surrounding the numerical superscript.

$[k]=k$ th basis function for the outer solution 
$(j)=j$ th term in the iteration series for a given basis function

$\{i\}=i$ th building block, corresponding to a prescribed seepage-velocity

or shear-stress profile along the permeable boundary.

In summary, one or more building blocks $\{i\}$ figure in each term $(j)$ of the iteration series representing a given basis function $[k]$ for the outer solution.

\subsection{Building blocks for the outer solution}

Eight specific solutions of (1.6)-(1.10), distinguished with numerical superscripts enclosed between curly brackets, serve as building blocks for the outer solution. These solutions do not, on an individual basis, satisfy the seepage condition (1.11). Rather, they are defined below by a hierarchy of seepage profiles $v_{\theta}^{\{i\}}(r, 0)$ along the permeable membrane boundary. The corresponding pressure and shear-stress profiles are as follows:

$$
\begin{gathered}
v_{\theta}^{\{1\}}(r, 0)=-1 \quad p^{\{1\}}(r, 0)=1-2 \pi^{-1} r^{-1} \\
v_{\theta}^{\{2\}}(r, 0)=r^{-1} \quad p^{\{2\}}(r, 0)=2 \pi^{-1} r^{-2}(1-\ln r) \\
v_{\theta}^{\{3\}}(r, 0)=0 \quad p^{\{3\}}(r, 0)=2 \pi^{-1} r^{-2} \\
v_{\theta}^{\{4\}}(r, 0)=0 \quad p^{\{4\}}(r, 0)=0 \\
v_{\theta}^{\{5\}}(r, 0)=r^{-2} \quad p^{\{5\}}(r, 0)=-\pi^{-1} r^{-3}(4 \ln r+5 / 3) \\
v_{\theta}^{\{6\}}(r, 0)=r^{-2} \ln r \quad p^{\{6\}}(r, 0)=\pi^{-1} r^{-3}\left[1+2 \ln r-2(\ln r)^{2}\right] \\
v_{\theta}^{\{7\}}(r, 0)=0 \quad p^{\{7\}}(r, 0)=0 \\
v_{\theta}^{\{8\}}(r, 0)=0 \quad p^{\{8\}}(r, 0)=2 r^{-3} \\
v_{r}^{\{1\}}(r, 0)=0 \quad \tau_{\theta r}^{\{1\}}(r, 0)=0 \\
v_{r}^{\{2\}}(r, 0)=0 \quad \tau_{\theta r}^{\{2\}}(r, 0)=-2 r^{-2} \\
v_{r}^{\{3\}}(r, 0)=0 \quad \tau_{\theta r}^{\{3\}}(r, 0)=0 \\
v_{r}^{\{4\}}(r, 0)=0 \quad \tau_{\theta r}^{\{4\}}(r, 0)=-4 \pi^{-1} r^{-2} \\
v_{r}^{\{5\}}(r, 0)=0 \quad \tau_{\theta r}^{\{5\}}(r, 0)=-4 r^{-3} \\
v_{\theta}^{\{6\}}(r, 0)=0 \quad \tau_{\theta r}^{\{6\}}(r, 0)=(11 / 3) r^{-3} \\
v_{r}^{\{7\}}(r, 0)=0 \quad \tau_{\theta r}^{\{7\}}(r, 0)=2 r^{-3} \\
v_{r}^{\{8\}}(r, 0)=0 \quad \tau_{\theta r}^{\{8\}}(r, 0)=0 .
\end{gathered}
$$

The first building block is the well-known solution (1.14)-(1.17) for a discontinuous jump from zero to unit seepage velocity along the wall (Taylor 1960, 1962; Moffatt 1964a; Pan \& Acrivos 1967; Hancock et al. 1981; Gupta et al. 1981; Harper \& Wake 1983; Nitsche \& Zhuge 1995). For the remaining solutions $(i=2, \ldots, 8)$, appendix C provides explicit formulas for the velocity and pressure fields in Cartesian coordinates. Here we are satisfied to summarize the general forms of the radial decay:

$\begin{array}{rcl}\text { Solution(s) } & \text { Velocity terms } & \text { Pressure terms } \\ \{1\} & 1 & 1, r^{-1} \\ \{2\} & r^{-1}, r^{-1} \ln r & r^{-2}, r^{-2} \ln r \\ \{3\},\{4\} & r^{-1} & r^{-2} \\ \{5\} & r^{-2}, r^{-2} \ln r & r^{-3}, r^{-3} \ln r\end{array}$




$$
\begin{aligned}
& \{6\} r^{-2}, r^{-2} \ln r, r^{-2}(\ln r)^{2} r^{-3}, r^{-3} \ln r, r^{-3}(\ln r)^{2} \\
& \{7\},\{8\} \quad r^{-2} .
\end{aligned}
$$

\subsection{Iteration on the seepage condition}

For a fixed outer domain $r>R$ we posit an asymptotic series

$$
\begin{aligned}
& \boldsymbol{v}(\boldsymbol{r})=\boldsymbol{v}^{(0)}(\boldsymbol{r})+\boldsymbol{v}^{(1)}(\boldsymbol{r})+\boldsymbol{v}^{(2)}(\boldsymbol{r})+\cdots \\
& p(\boldsymbol{r})=p^{(0)}(\boldsymbol{r})+p^{(1)}(\boldsymbol{r})+p^{(2)}(\boldsymbol{r})+\cdots
\end{aligned}
$$

in which the ascending terms are obtained by iteration on the seepage condition (1.11). The original governing equations (1.6)-(1.10) apply to each flow field $\left\{\boldsymbol{v}^{(j)}(\boldsymbol{r}), p^{(j)}(\boldsymbol{r})\right\}$. However, the seepage condition (1.11) is altered so that the seepage flux at each order is now driven by the pressure at the previous order:

$$
v_{\theta}^{(j)}(r, 0)=-p^{(j-1)}(r, 0) .
$$

In view of the far-field conditions (1.12) and (1.13), it is natural to start off the iteration with the well-known similarity solution (1.14)-(1.16).

$$
\boldsymbol{v}^{(0)} \equiv \boldsymbol{v}^{\{1\}}, \quad p^{(0)} \equiv p^{\{1\}} .
$$

The constant term in the wall pressure profile $p^{\{1\}}(r, 0)$ is balanced by the uniform seepage velocity $v_{\theta}^{\{1\}}(r, 0)$; cf. equations (1.11) and (4.4). The remaining $\operatorname{ord}\left(r^{-1}\right)$ wall pressure term then drives a new seepage velocity according to the shifted-order seepage condition (4.28). This is supplied by a suitable multiple of (4.5) at first order. From (4.13) we know that this will be accompanied by a non-zero shear stress along the membrane, which can be cancelled out by subtracting the corresponding contribution from (4.15) while leaving the pressure and seepage velocity untouched according to (4.7):

$$
\boldsymbol{v}^{(1)} \equiv(2 / \pi) \boldsymbol{v}^{\{2\}}-\boldsymbol{v}^{\{4\}}, \quad p^{(1)} \equiv(2 / \pi) p^{\{2\}}-p^{\{4\}} .
$$

Now we have two terms in the membrane pressure profile $p^{(1)}(r, 0)$, namely $(2 / \pi)^{2} r^{-2}$ and $-(2 / \pi)^{2} r^{-2} \ln r$. These are matched by the respective seepage velocities in (4.8) and (4.9). The resultant shear stress from (4.16) and (4.17) can be cancelled out by subtracting a suitable multiple of the corresponding contribution from (4.18), while again leaving the pressure and seepage velocity untouched according to (4.10):

$$
\begin{aligned}
& \boldsymbol{v}^{(2)} \equiv\left(\frac{2}{\pi}\right)^{2}\left[\boldsymbol{v}^{\{6\}}-\boldsymbol{v}^{\{5\}}-\left(\frac{23}{6}\right) \boldsymbol{v}^{\{7\}}\right] \\
& p^{(2)} \equiv\left(\frac{2}{\pi}\right)^{2}\left[p^{\{6\}}-p^{\{5\}}-\left(\frac{23}{6}\right) p^{\{7\}}\right] .
\end{aligned}
$$

\subsection{Basis functions for the outer solution}

To summarize results of the above iterative procedure, the well-known similarity solution (1.14)-(1.16) starts off one perturbation series

$$
\begin{aligned}
\boldsymbol{v}^{[0]}(\boldsymbol{r})= & \boldsymbol{v}^{\{1\}}(\boldsymbol{r})+\left(\frac{2}{\pi}\right)\left[\boldsymbol{v}^{\{2\}}(\boldsymbol{r})-\left(\frac{\pi}{2}\right) \boldsymbol{v}^{\{4\}}(\boldsymbol{r})\right] \\
& +\left(\frac{2}{\pi}\right)^{2}\left[\boldsymbol{v}^{\{6\}}(\boldsymbol{r})-\boldsymbol{v}^{\{5\}}(\boldsymbol{r})-\left(\frac{23}{6}\right) \boldsymbol{v}^{\{7\}}(\boldsymbol{r})\right]+O\left[r^{-3}(\ln r)^{3}\right]
\end{aligned}
$$




$$
\begin{aligned}
p^{[0]}(\boldsymbol{r})= & p^{\{1\}}(\boldsymbol{r})+\left(\frac{2}{\pi}\right)\left[p^{\{2\}}(\boldsymbol{r})-\left(\frac{\pi}{2}\right) p^{\{4\}}(\boldsymbol{r})\right] \\
& +\left(\frac{2}{\pi}\right)^{2}\left[p^{\{6\}}(\boldsymbol{r})-p^{\{5\}}(\boldsymbol{r})-\left(\frac{23}{6}\right) p^{\{6\}}(\boldsymbol{r})\right]+O\left[r^{-4}(\ln r)^{3}\right]
\end{aligned}
$$

which represents a fixed basis function for the outer solution. This Stokes solution satisfies both the no-slip condition (1.10) and the no-shear condition

$$
\tau_{\theta r}(r, 0)=0 .
$$

Two other solutions cannot be excluded because their pressure and velocity fields vanish at infinity.

$$
\begin{gathered}
\boldsymbol{v}^{[1]}(\boldsymbol{r})=\boldsymbol{v}^{\{3\}}(\boldsymbol{r})-\left(\frac{2}{\pi}\right)\left[\boldsymbol{v}^{\{5\}}(\boldsymbol{r})+2 \boldsymbol{v}^{\{7\}}(\boldsymbol{r})\right] \\
\boldsymbol{v}^{[2]}(\boldsymbol{r})=\boldsymbol{v}^{\{8\}}(\boldsymbol{r}) .
\end{gathered}
$$

These should be included with floating coefficients to be determined by matching to the inner solution.

\section{Numerical solution patched to the outer asymptotics}

The basis functions (4.33), (4.34), (4.36), (4.37) from $\$ 4.3$ provide us with a second-order-accurate outer field for reformulating the boundary conditions (1.12), (1.13) at finite radius $(r=30)$ :

$$
\begin{aligned}
& \boldsymbol{v}^{\text {outer }}\left(30, \theta ; C_{1}, C_{2}\right)=\boldsymbol{v}^{[0]}(30, \theta)+\sum_{k=1}^{2} C_{k} \boldsymbol{v}^{[k]}(30, \theta) \\
& p^{\text {outer }}\left(30, \theta ; C_{1}, C_{2}\right)=p^{[0]}(30, \theta)+\sum_{k=1}^{2} C_{k} p^{[k]}(30, \theta) .
\end{aligned}
$$

Armed with these formulas, we expect to overcome limitations of our preliminary numerical solution $(\$ 2.2)$ that were evident in figures 4-6.

\subsection{Iterative patching for the outer boundary condition}

A slight complication arises in using the outer field (5.1), (5.2): the coefficients $C_{1}$ and $C_{2}$ of the floating terms are not known a priori. These represent inputs to the inner solution, but are themselves (in theory) also determined by the inner behaviour. A rigorous asymptotic approach would involve matching of inner and outer expansions over the common range of validity of an intermediate radial variable. However, even carried through second order, the inner asymptotic solution (to be developed in $\S 6$ ) will not extend far enough toward the outer asymptotic solution; see figures 11 and 12. Thus, we shall proceed here with an iterative scheme to patch an inner numerical solution to the outer asymptotic solution along the arc $r=30$; see figure 2 .

We begin by setting both floating coefficients in (5.1) and (5.2) equal to zero, and prescribe outer boundary conditions for $v_{\theta}(30, \theta)$ and $p(30, \theta)$ according to only the $a$ priori known portion of the second-order outer solution: namely, $v_{\theta}^{[0]}$ and $p^{[0]}$. Along the semicircular boundary $r=30$, the numerically calculated radial velocity $v_{r}^{\text {numer }}(30, \theta)$, which was not prescribed with a boundary condition, will initially not be consistent with $v_{r}^{\text {outer }}(30, \theta ; 0,0)$. We then carry out a linear, least-squares fit of the 


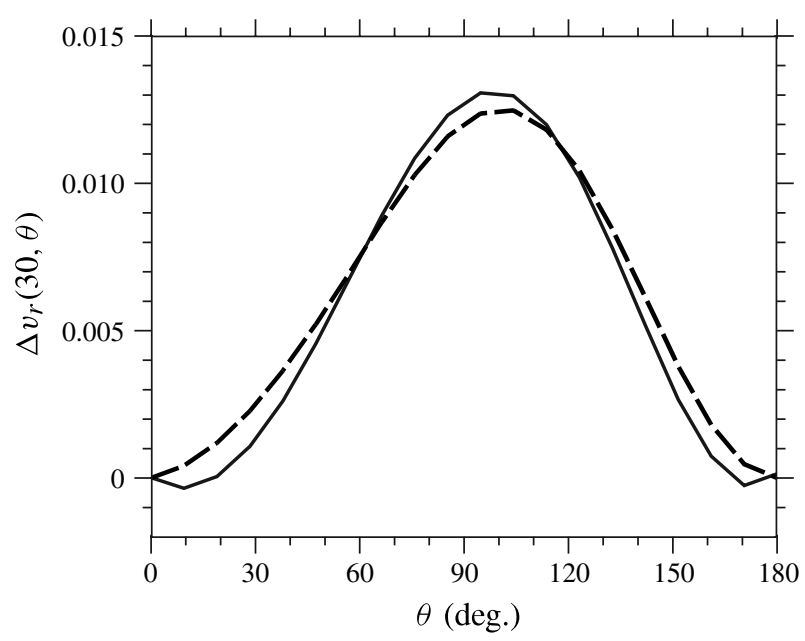

FIGURE 8. Solid line: initial discrepancy between the numerically calculated radial velocity component and the outer asymptotic solution. Dashed line: a least-squares fit using both free coefficients in (5.1).

discrepancy

$$
\Delta v_{r}(30, \theta) \stackrel{\text { def }}{=} v_{r}^{\text {numer }}(30, \theta)-v_{r}^{\text {outer }}(30, \theta ; 0,0)
$$

using as basis functions the two floating solutions $v_{r}^{[1]}$ and $v_{r}^{[2]}$ from (5.1) regarded as functions of $\theta$ at fixed $r=30$; see figure 8 .

The resulting updated coefficients $C_{1}$ and $C_{2}$ are fed back into (5.1) and (5.2) to prescribe outer boundary conditions for the next iteration of the numerical scheme. Since the least-squares matrix has already been factorized, only a quick back substitution is needed to obtain singularity strengths for the new right-hand side (updated conditions at the outer boundary points). The converged constants appearing in (5.1) and (5.2) are as follows:

$$
C_{1}=-0.5719, \quad C_{2}=1.6895
$$

Figures 10-12 show the iteration scheme to yield a very smooth patch of the numerical solution to the second-order outer solution at $r=30$, much better than was obtained with the zero-order patching scheme (figures 4-6).

\section{Inner asymptotic solution}

Uniform seepage along the porous boundary $(\theta=0)$ involves a jump discontinuity in the normal velocity component, which is associated with an $r^{-1}$ singularity in the pressure in (1.14). From the numerical results depicted in figures 7 and 9 we see that seepage flux proportional to the transmembrane pressure drop, (1.11), allows the normal velocity component to remain continuous at the origin while also approaching the horizontal asymptote of -1 as $r \rightarrow \infty$. This partly alleviates the singularity, so that now the pressure only blows up like $r^{-1 / 2}$ as $r \rightarrow 0$; see figure 7 . 


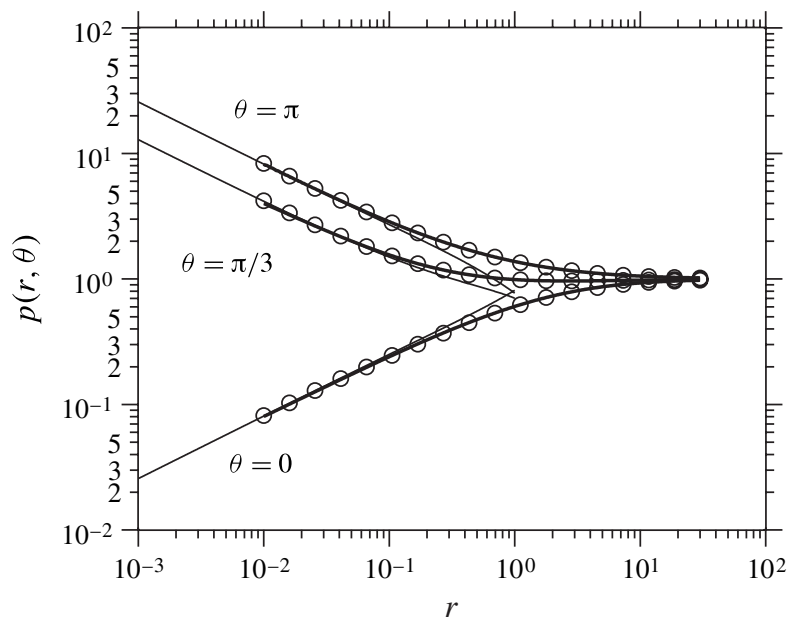

FIGURE 9. Numerically calculated pressure plotted as a function of radius $r$ for $\theta=0, \pi / 3, \pi$ (thick lines). Thin lines indicate the inner asymptotic solution (6.12). Open circles indicate the polynomial fit described in $\S 7$.

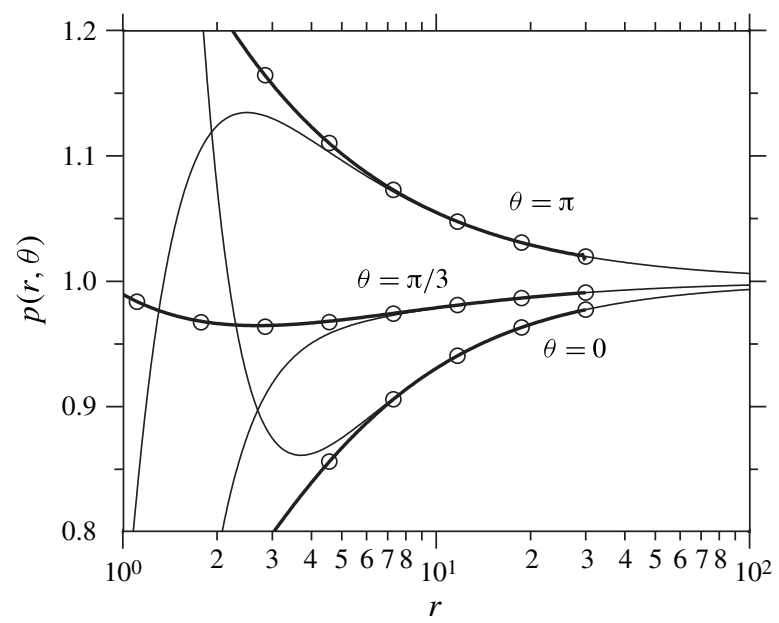

FIgURE 10. Detail view of the pressure profiles from figure 9. The numerical curves (thick) merge smoothly with the outer asymptotic curves (thin) that specify the boundary conditions at $r=30$. Open circles indicate the polynomial fit described in $\S 7$.

\subsection{Leading similarity solution}

Specializing the general formulas in $\S 3$ to the case of a similarity solution (namely, $F \equiv 0$ and $G \equiv 0)$, we consider the half-integral values $\omega=n+(1 / 2)$ :

$$
\begin{aligned}
v_{r}^{(n)}(r, \theta)= & {\left[A_{n}\left\{\left(n-\frac{1}{2}\right) \sin \left[\left(n-\frac{1}{2}\right) \theta\right]-\left(n-\frac{1}{2}\right) \sin \left[\left(n+\frac{3}{2}\right) \theta\right]\right\}\right.} \\
& \left.-B_{n}\left\{\left(n-\frac{1}{2}\right) \cos \left[\left(n-\frac{1}{2}\right) \theta\right]-\left(n+\frac{3}{2}\right) \cos \left[\left(n+\frac{3}{2}\right) \theta\right]\right\}\right] r^{n+1 / 2} \\
v_{\theta}^{(n)}(r, \theta)= & {\left[A_{n}\left\{\left(n+\frac{3}{2}\right) \cos \left[\left(n-\frac{1}{2}\right) \theta\right]-\left(n-\frac{1}{2}\right) \cos \left[\left(n+\frac{3}{2}\right) \theta\right]\right\}\right.} \\
& \left.+B_{n}\left\{\left(n+\frac{3}{2}\right) \sin \left[\left(n-\frac{1}{2}\right) \theta\right]-\left(n+\frac{3}{2}\right) \sin \left[\left(n+\frac{3}{2}\right) \theta\right]\right\}\right] r^{n+1 / 2}
\end{aligned}
$$




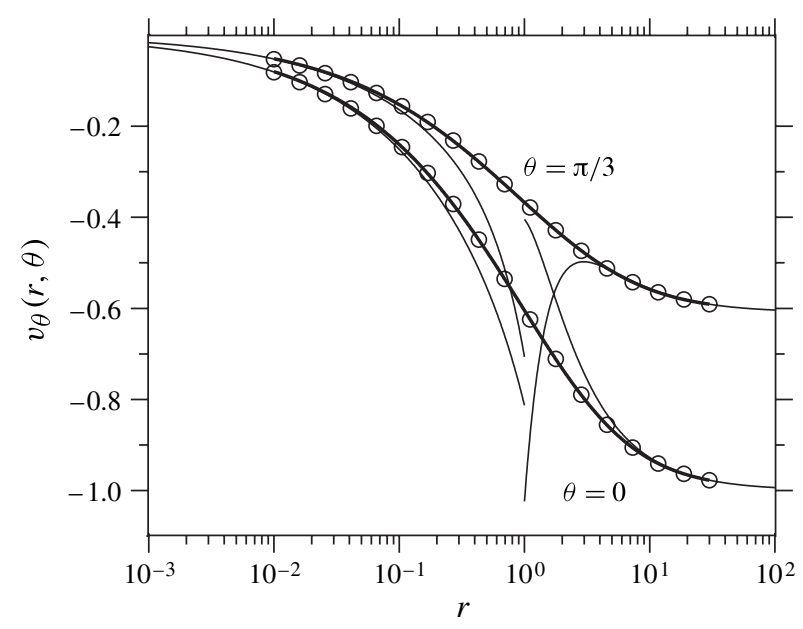

FIGURE 11. Numerically calculated angular velocity component plotted as a function of radius $r$ for $\theta=0, \pi / 3$ (thick lines). Thin lines indicate the inner (6.11) and outer (5.1) asymptotic solutions. Open circles indicate the polynomial fit described in $\S 7$.

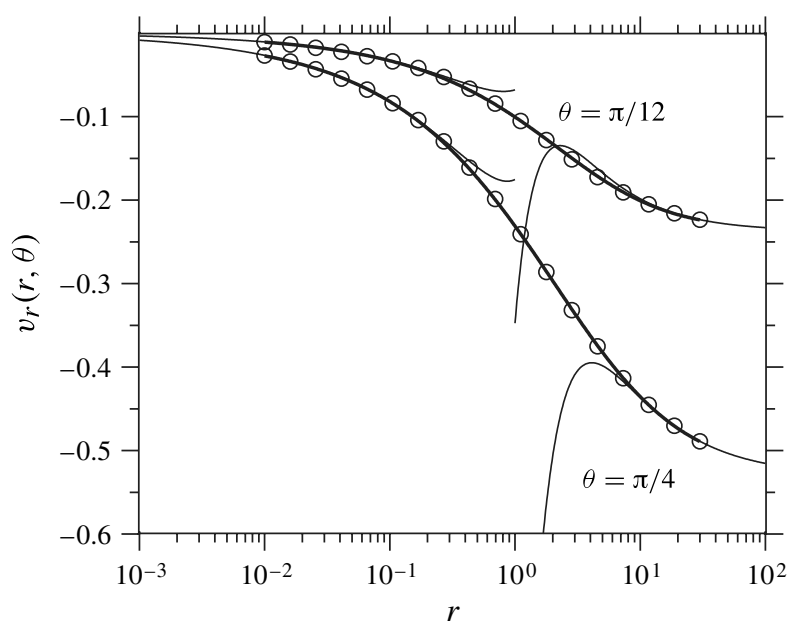

FIGURE 12. Numerically calculated radial velocity component plotted as a function of radius $r$ for $\theta=\pi / 12, \pi / 4$ (thick lines). Thin lines indicate the inner (6.10) and outer (5.1) asymptotic solutions. Open circles indicate the polynomial fit described in $\S 7$.

$$
p^{(n)}(r, \theta)=4\left(n+\frac{1}{2}\right)\left\{A_{n} \sin \left[\left(n-\frac{1}{2}\right) \theta\right]-B_{n} \cos \left[\left(n-\frac{1}{2}\right) \theta\right]\right\} r^{n-1 / 2} .
$$

This flow field satisfies the no-slip (1.8) and impermeability (1.9) conditions along the solid wall $(\theta=\pi)$. Along the membrane boundary $(\theta=0)$ we have

$$
\begin{gathered}
v_{r}^{(n)}(r, 0)=2 B_{n} r^{n+1 / 2} \\
v_{\theta}^{(n)}(r, 0)=2 A_{n} r^{n+1 / 2} \\
p^{(n)}(r, 0)=-4 B_{n}\left(n+\frac{1}{2}\right) r^{n-1 / 2} .
\end{gathered}
$$


Let us start with $n=0$. Setting $B_{0}=0$ eliminates slip and keeps the pressure finite along the membrane, according to (6.4) and (6.6). Choosing $A_{0}=-1$, the seepage velocity profile $v_{\theta}^{(0)}(r, 0)=-2 r^{1 / 2}$ from (6.5) must be driven by the pressure field $p^{(1)}(r, 0)$ with $B_{1}=-1 / 3$. Setting $A_{1}=0$ then eliminates any further discrepancy between the seepage flux and the driving pressure drop. Unfortunately, (6.4) now shows an error in the no-slip condition due to the non-zero value of $B_{1}$ required in the pressure field. We cannot come beyond this impasse with solutions of the similarity form. Nevertheless, we have the leading inner asymptotic flow field

$$
\begin{aligned}
& v_{r}(r, \theta) \sim-\left\{\frac{1}{2} \sin \left(\frac{\theta}{2}\right)+\frac{1}{2} \sin \left(\frac{3 \theta}{2}\right)\right\} r^{1 / 2} \\
&+\left\{\frac{1}{6} \cos \left(\frac{\theta}{2}\right)-\frac{5}{6} \cos \left(\frac{5 \theta}{2}\right)\right\} r^{3 / 2} \\
& v_{\theta}(r, \theta) \sim-\left\{\frac{3}{2} \cos \left(\frac{\theta}{2}\right)+\frac{1}{2} \cos \left(\frac{3 \theta}{2}\right)\right\} r^{1 / 2} \\
&-\left\{\frac{5}{6} \sin \left(\frac{\theta}{2}\right)-\frac{5}{6} \sin \left(\frac{5 \theta}{2}\right)\right\} r^{3 / 2} \\
& p(r, \theta) \sim\left\{2 \sin \left(\frac{\theta}{2}\right)\right\} r^{-1 / 2}+\left\{2 \cos \left(\frac{\theta}{2}\right)\right\} r^{1 / 2}
\end{aligned}
$$

because the ord $\left(r^{3 / 2}\right)$ error in the slip condition is asymptotically negligible compared with the $\operatorname{ord}\left(r^{1 / 2}\right)$ magnitude of the velocity. This result was presented in the dissertation of Parthasarathi (2008).

\subsection{Second-order inner solution involving logarithmic terms}

The impasse in the no-slip condition can be obviated if we are willing to entertain logarithmic terms from $\S 3$. The iterative approach whereby ascending terms cancel previous errors in the boundary conditions will be familiar from $\S 4.2$, so we shall here dispense with details and present only the final result. With reference to the general solution (B 1)-(B 4) from appendix B, we find

$$
\begin{aligned}
& v_{r}^{\text {inner }}(r, \theta)=C \sum_{n=1}^{4} v_{r}\left(r, \theta ; n-1 / 2, \boldsymbol{a}^{\{n\}}, \boldsymbol{b}^{\{n\}}, \boldsymbol{c}^{[n\}}\right) \\
& v_{\theta}^{\text {inner }}(r, \theta)=C \sum_{n=1}^{4} v_{\theta}\left(r, \theta ; n-1 / 2, \boldsymbol{a}^{\{n\}}, \boldsymbol{b}^{\{n\}}, \boldsymbol{c}^{\{n\}}\right) \\
& p^{\text {inner }}(r, \theta)=C \sum_{n=1}^{4} p\left(r, \theta ; n-1 / 2, \boldsymbol{a}^{\{n\}}, \boldsymbol{b}^{\{n\}}, \boldsymbol{c}^{\{n\}}\right) \\
& \tau_{\theta r}^{\text {inner }}(r, \theta)=C \sum_{n=1}^{4} \tau_{\theta r}\left(r, \theta ; n-1 / 2, \boldsymbol{a}^{\{n\}}, \boldsymbol{b}^{\{n\}}, \boldsymbol{c}^{\{n\}}\right)
\end{aligned}
$$

with the relevant constants appearing in table 1 . This Stokes flow field exactly satisfies the no-slip (1.8) and no-penetration (1.9) conditions along the impermeable boundary $(\theta=\pi)$ as well as the seepage condition (1.11) along the porous membrane $(\theta=0)$. Along the latter boundary the tangential velocity and shear stress are asymptotically small, i.e. of third order relative to the leading velocity $\left(r^{1 / 2}\right)$ and pressure $\left(r^{-1 / 2}\right)$ 


$$
\begin{aligned}
& \begin{array}{l}
t \\
0
\end{array} \\
& x \times x \times x
\end{aligned}
$$

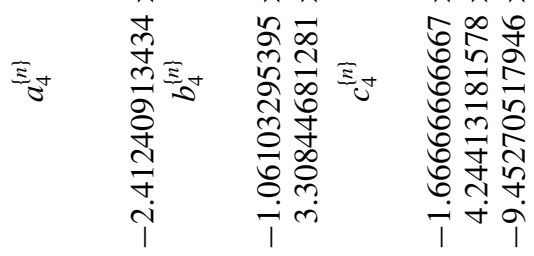

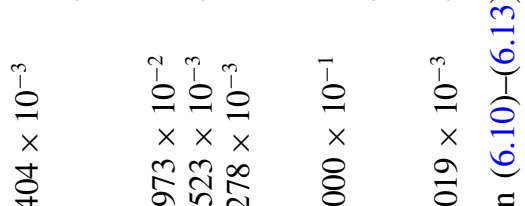

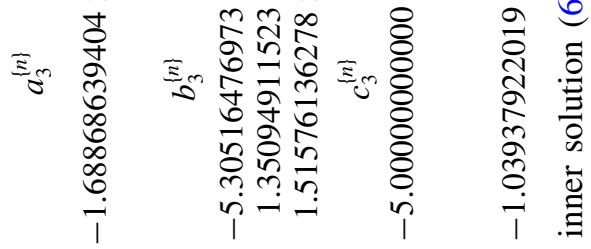

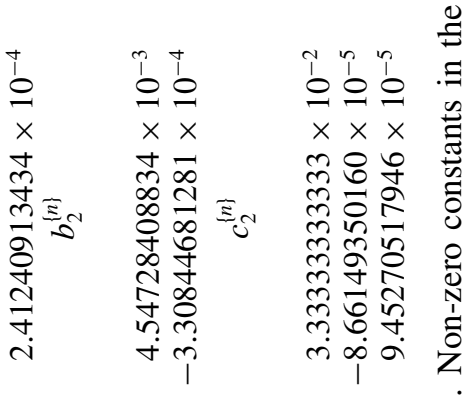

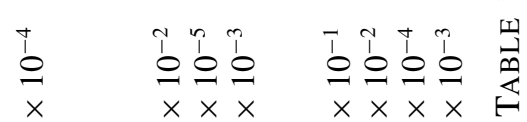

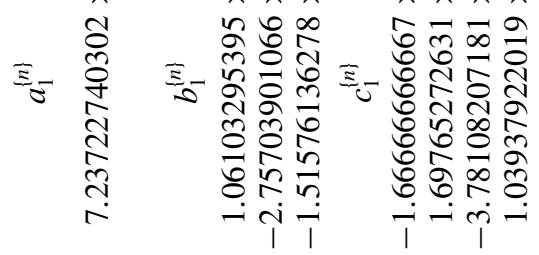

$$
\begin{aligned}
& =m+=N m \theta=-N m \theta
\end{aligned}
$$




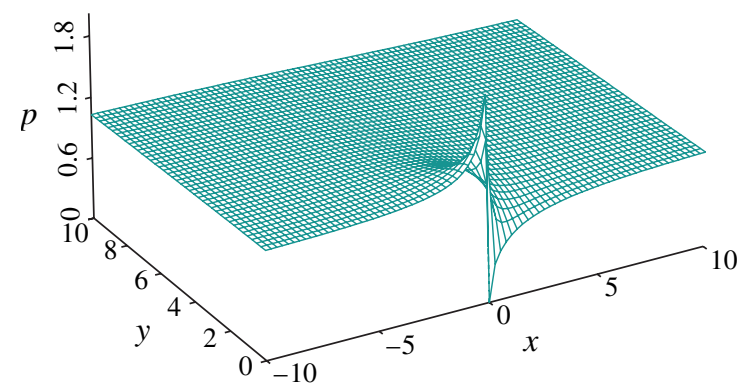

FIGURE 13. (Colour online) Surface plot of $p(x, y)$.

terms:

$$
v_{r}(r, 0)=O\left[r^{7 / 2}(\ln r)^{2}\right], \quad \tau_{\theta r}(r, 0)=O\left[r^{5 / 2} \ln r\right] .
$$

Since slip velocity and shear stress both vanish within negligible errors, the Beavers-Joseph slip condition (Beavers \& Joseph 1967; Jones 1973; Nield 1983) is thereby satisfied as well; see $\S 1$ of the supplementary material.

\section{A uniformly valid, analytical formula}

The numerical velocity components and pressure, regarded as functions of $\theta$ at $r=0.01$, yield the prefactor for the inner asymptotic solution (6.10)-(6.13):

$$
C=0.8128 \text {. }
$$

We shall use this solution for $r<0.01$ and the outer asymptotic solution (5.1), (5.2), (5.4) for $r>30$. To bridge the gap at any fixed value of $\theta$, we approximate $\ln p(r, \theta), v_{r}(r, \theta)$ and $v_{\theta}(r, \theta)$ with eighth-degree polynomials in the variable $\ln r$, whose coefficients are chosen to match: (i) the asymptotic function values and first two derivatives with respect to $\ln r$ at the ends; and (ii) the numerical function values at three tabulated intermediate radii approximately equal to $r=0.1,1,10$, respectively. The latter three functions of $\theta$ at fixed $r$ are obtained by fitting cubic splines to the numerical data at angular increments $\Delta \theta=\pi / 12$.

The polynomial fits approximate the numerical solution very accurately, as can be seen in figures 9-12. Figures 13-15 show plots of $p(x, y), v_{x}(x, y)$ and $v_{y}(x, y)$ based upon the uniformly valid fit.

\section{Concluding remarks}

An abrupt transition between impermeable and permeable portions of a planar wall is typical of the upstream section of a reverse-osmosis module (Miranda \& Campos 2001; Geraldes et al. 2002b). This paper has resolved the two-dimensional Stokes flow singularity found at such special points. In particular, the pressure field has an integrable singularity (of order $r^{-1 / 2}$ ), and the seepage velocity is continuous (scaling like $r^{1 / 2}$ ). By contrast, assuming a uniform seepage velocity that jumps discontinuously to zero at the junction with the impermeable section leads to a non-integrable, $r^{-1}$ divergence in pressure. Such details are important to quantify because: (i) reverse osmosis is sensitive to the local transmembrane pressure drop; and (ii) the upstream zone, where the concentration-polarization boundary layer is not yet fully developed, is the most productive portion of the membrane. 


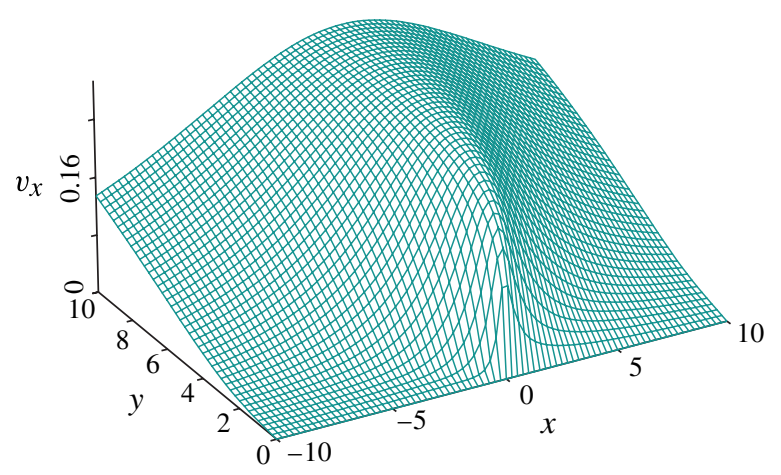

FIGURE 14. (Colour online) Surface plot of $v_{x}(x, y)$.

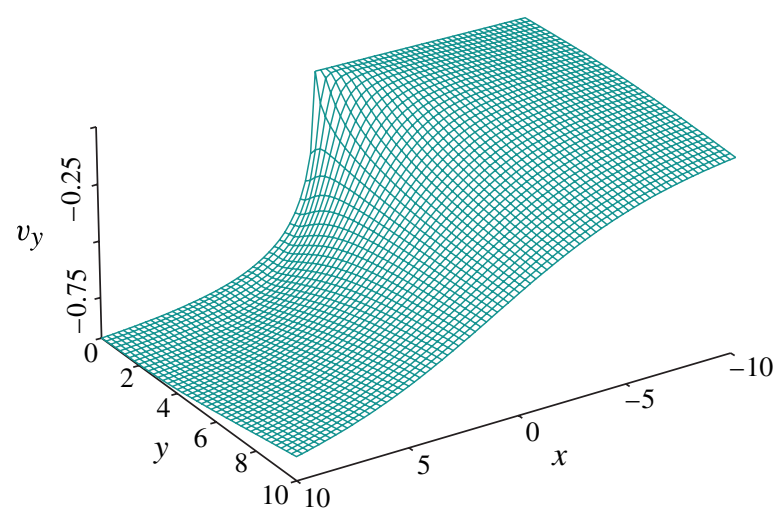

FIGURE 15. (Colour online) Surface plot of $v_{y}(x, y)$.

A major theme of this paper has been the fruitful interplay between numerical and asymptoptic analysis, both of which were needed to solve the fluid mechanical problem at hand. Initial numerics guided the inner asymptotics by indicating the radial scaling of the flow singularity, but suffered from poor satisfaction of the outer boundary conditions at $r=30$. Consistency for these boundary conditions was dramatically improved using a hybrid numerical-asymptotic scheme. Slowly decaying, logarithmic terms in the outer asymptotic expansion explain why a purely numerical approach would require a much larger domain, over most of which the analytical formulas would yield indistinguishable results with much less computation time. The inner asymptotic expansion allows the singular fine structure to be resolved at arbitrarily small $r$ without the need for extreme mesh refinement. The numerics (as fitted with suitable polynomials in $\ln r$ ) bridge between the inner and outer asymptotics.

To maximize the usability of our results for readers who may wish to use the formulas in their own calculations, $\S 4$ of the supplementary material contains a Fortran 2003 library that evaluates: (i) the general solution (B 1)-(B 4) from appendix B for arbitrary radial exponent $\omega$, including the special cases $\omega= \pm 1$; and (ii) the uniformly valid porous-wall solution described in $\S 7$; see figures 13-15. Such solutions could be used as local basis functions within a more extensive numerical 
scheme (Bruns 1979; Ingham \& Kelmanson 1984; Floryan \& Czechowski 1995; Nitsche \& Zhuge 1995; Hawa \& Rusak 2002; Shi et al. 2004; Shapeev \& Lin 2009), to reduce the need for local mesh refinement. Compared with local power series involving similarity solutions (Hawa \& Rusak 2002; Shi et al. 2004; Shapeev \& Lin 2009), the logarithmic solutions from $\S 3$ expand the palette of Stokes basis functions and go beyond the first power of $\ln r$ thus far considered in other papers (Sinclair et al. 2009; Sinclair 2010). By way of a posteriori validation, the Fortran code subjects the flow fields to discretized (i.e. finite-difference) versions of the continuity and Stokes equations, and also checks harmonicity of the pressure. Calling sequences for the relevant subroutines are illustrated with a short sample program in appendix D.

\section{Supplementary material}

Supplementary material is available at journals.cambridge.org/flm.

\section{Appendix A. Weakly singular, three-point basis function from $\S 2.1$}

Using a two-point, linearly ramped singularity distribution

$$
\boldsymbol{L}(\boldsymbol{r} ; \boldsymbol{a}, \boldsymbol{b}, m)=\|\boldsymbol{b}-\boldsymbol{a}\| \int_{0}^{1} s \boldsymbol{G}[\boldsymbol{r}-\boldsymbol{a}-s(\boldsymbol{b}-\boldsymbol{a})] \mathrm{d} s,
$$

we can rewrite (2.2) for the three-point singular basis function:

$$
\boldsymbol{F}(\boldsymbol{r} ; \boldsymbol{a}, \boldsymbol{b}, \boldsymbol{c})=\boldsymbol{L}(\boldsymbol{r} ; \boldsymbol{a}, \boldsymbol{b},-1)+\boldsymbol{L}(\boldsymbol{r} ; \boldsymbol{c}, \boldsymbol{b}, 1) .
$$

In (A 1) the variable $m$ indicates the infinitesimal displacement in the limit as the point of evaluation $\boldsymbol{r}$ approaches the directed line segment $\overrightarrow{\boldsymbol{a}} \overrightarrow{\mathrm{b}}$ :

$$
m= \begin{cases}-1 & \text { left } \\ 0 & \text { none (principal value) } \\ 1 & \text { right. }\end{cases}
$$

From (2.1) follows a simple relation between the pressure fields of the force basis functions and the velocity components of the source basis function:

$$
L_{0, i}=2 L_{i, 0} .
$$

We define relative position vectors $\zeta, \eta$ and $\lambda$

$$
\zeta=r-a, \quad \eta=r-b, \quad \lambda=b-a,
$$

along with the unit normal vector $n$

$$
\boldsymbol{n}=\|\boldsymbol{b}-\boldsymbol{a}\|^{-1}\left(b_{2}-a_{2}, a_{1}-b_{1}\right)
$$

and the coefficients

$$
A=\|\zeta\|^{2}, \quad B=\|\eta\|^{2}, \quad C=\|\lambda\|^{2}, \quad D=\zeta \cdot \lambda .
$$

The discriminant $A C-D^{2}$ is always non-negative, and it vanishes when the point $\boldsymbol{r}$ lies somewhere on the line passing through $\boldsymbol{a}$ and $\boldsymbol{b}$. In this eventuality the further restriction $0<D / C<1$ indicates the weakly singular limit wherein $\boldsymbol{r}$ lies between $\boldsymbol{a}$ and $\boldsymbol{b}$. Let

$$
E=\sqrt{A C-D^{2}}, \quad F=D / C .
$$


For the singular case $E=0$ (numerically, $E$ below some tolerance) we use the following formulas with $1 \leqslant i, j \leqslant 2$ :

$$
\begin{gathered}
L_{i, 0}=\left\{F \ln \left|\frac{F}{1-F}\right|-1\right\} \frac{\lambda_{i}}{C}+\{1+\operatorname{sgn}[F(1-F)]\} \frac{m \pi F n_{i}}{2 \sqrt{C}} \\
L_{i, j}=\left\{1+2 F-2 F^{2} \ln |F|-2\left(1-F^{2}\right) \ln |1-F|-\ln C\right\} \frac{\delta_{i j}}{4}+\frac{\lambda_{i} \lambda_{j}}{2 C} .
\end{gathered}
$$

Otherwise we have

$$
\begin{gathered}
L_{i, 0}=\frac{X}{E}\left\{\frac{D \zeta_{i}+A \lambda_{i}}{C}-\frac{2 D^{2} \lambda_{i}}{C^{2}}\right\}+Y\left\{\frac{\zeta_{i}}{2 C}-\frac{D \lambda_{i}}{C^{2}}\right\}-\frac{\lambda_{i}}{C} \\
L_{i, j}=R_{i, j}+Z \delta_{i j}
\end{gathered}
$$

with

$$
\begin{gathered}
X=\arctan (D / E)-\arctan [(D-C) / E] \\
Y=\ln (B / A) \\
Z=\frac{D}{2 C}+Y\left(\frac{D^{2}}{2 C^{2}}-\frac{A}{4 C}\right)-\frac{D E X}{C^{2}}+\frac{1-\ln B}{4} \\
P_{i, j}=\frac{4 D^{2} \lambda_{i} \lambda_{j}}{C^{3}}+\frac{\zeta_{i} \zeta_{j}}{C}-\frac{2 D\left(\zeta_{i} \lambda_{j}+\zeta_{j} \lambda_{i}\right)+A \lambda_{i} \lambda_{j}}{C^{2}} \\
Q_{i, j}=\frac{A\left(\zeta_{i} \lambda_{j}+\zeta_{j} \lambda_{i}\right)}{C^{2}}-\frac{2 A D \lambda_{i} \lambda_{j}}{C^{3}} \\
R_{i, j}=\frac{Y P_{i, j}}{2}+\frac{X\left(D P_{i, j}+C Q_{i, j}\right)}{E}-\frac{\zeta_{i} \lambda_{j}+\zeta_{j} \lambda_{i}}{C}+\left(\frac{1}{2 C}+\frac{2 D}{C^{2}}\right) \lambda_{i} \lambda_{j} .
\end{gathered}
$$

\section{Appendix B. General solution from $\S 3$}

Equation (3.3) gave the stream function as a power law in $r$ multiplied by a quadratic polynomial in $\ln r$. The coefficients in the latter were functions of $\theta$. When the general solutions for these, (3.16)-(3.18), are substituted into the formulas (3.10)-(3.13) for the velocity, pressure and shear stress, we obtain

$$
\begin{aligned}
v_{r}(r, \theta ; \omega, \boldsymbol{a}, \boldsymbol{b}, \boldsymbol{c})= & \left\{\left[\boldsymbol{a} \cdot \boldsymbol{f}_{r}^{\{a 2\}}(\theta ; \omega)\right](\ln r)^{2}+\left[\boldsymbol{a} \cdot \boldsymbol{f}_{r}^{\{a 1\}}(\theta ; \omega)+\boldsymbol{b} \cdot \boldsymbol{f}_{r}^{\{b 1\}}(\theta ; \omega)\right] \ln r\right. \\
& \left.+\left[\boldsymbol{a} \cdot \boldsymbol{f}_{r}^{\{a 0\}}(\theta ; \omega)+\boldsymbol{b} \cdot \boldsymbol{f}_{r}^{\{b 0\}}(\theta ; \omega)+\boldsymbol{c} \cdot \boldsymbol{f}_{r}^{\{c 0\}}(\theta ; \omega)\right]\right\} r^{\omega} \\
v_{\theta}(r, \theta ; \omega, \boldsymbol{a}, \boldsymbol{b}, \boldsymbol{c})= & \left\{\left[\boldsymbol{a} \cdot \boldsymbol{f}_{\theta}^{\{a 2\}}(\theta ; \omega)\right](\ln r)^{2}+\left[\boldsymbol{a} \cdot \boldsymbol{f}_{\theta}^{\{a 1\}}(\theta ; \omega)+\boldsymbol{b} \cdot \boldsymbol{f}_{\theta}^{\{b 1\}}(\theta ; \omega)\right] \ln r\right. \\
& \left.+\left[\boldsymbol{a} \cdot \boldsymbol{f}_{\theta}^{\{a 0\}}(\theta ; \omega)+\boldsymbol{b} \cdot \boldsymbol{f}_{\theta}^{\{b 0\}}(\theta ; \omega)+\boldsymbol{c} \cdot \boldsymbol{f}_{\theta}^{\{c 0\}}(\theta ; \omega)\right]\right\} r^{\omega} \\
p(r, \theta ; \omega, \boldsymbol{a}, \boldsymbol{b}, \boldsymbol{c})= & \left\{\left[\boldsymbol{a} \cdot \boldsymbol{f}_{p}^{\{a 2\}}(\theta ; \omega)\right](\ln r)^{2}+\left[\boldsymbol{a} \cdot \boldsymbol{f}_{p}^{\{a 1\}}(\theta ; \omega)+\boldsymbol{b} \cdot \boldsymbol{f}_{p}^{\{b 1\}}(\theta ; \omega)\right] \ln r\right. \\
& \left.+\left[\boldsymbol{a} \cdot \boldsymbol{f}_{p}^{\{a 0\}}(\theta ; \omega)+\boldsymbol{b} \cdot \boldsymbol{f}_{p}^{\{b 0\}}(\theta ; \omega)+\boldsymbol{c} \cdot \boldsymbol{f}_{p}^{\{c 0\}}(\theta ; \omega)\right]\right\} r^{\omega-1} \\
\tau_{\theta r}(r, \theta ; \omega, \boldsymbol{a}, \boldsymbol{b}, \boldsymbol{c})= & \left\{\left[\boldsymbol{a} \cdot \boldsymbol{f}_{\tau}^{\{a 2\}}(\theta ; \omega)\right](\ln r)^{2}+\left[\boldsymbol{a} \cdot \boldsymbol{f}_{\tau}^{\{a 1\}}(\theta ; \omega)+\boldsymbol{b} \cdot \boldsymbol{f}_{\tau}^{\{b 1\}}(\theta ; \omega)\right] \ln r\right. \\
& \left.+\left[\boldsymbol{a} \cdot \boldsymbol{f}_{\tau}^{\{a 0\}}(\theta ; \omega)+\boldsymbol{b} \cdot \boldsymbol{f}_{\tau}^{\{b 0\}}(\theta ; \omega)+\boldsymbol{c} \cdot \boldsymbol{f}_{\tau}^{\{c 0\}}(\theta ; \omega)\right]\right\} r^{\omega-1}
\end{aligned}
$$

with

$$
\begin{gathered}
\boldsymbol{f}_{r}^{\{a 2\}}(\theta ; \omega)=\boldsymbol{f}_{r}^{\{b 1\}}(\theta ; \omega)=\boldsymbol{f}_{r}^{\{c 0\}}(\theta ; \omega)=-\lambda_{1}(\theta ; \omega) \\
\boldsymbol{f}_{r}^{\{a 1\}}(\theta ; \omega)=2 \theta \boldsymbol{\kappa}_{1}(\theta ; \omega)-2 \boldsymbol{\lambda}_{0}(\theta ; \omega) \\
\boldsymbol{f}_{r}^{\{a 0\}}(\theta ; \omega)=2 \theta \boldsymbol{\kappa}_{0}(\theta ; \omega)+\theta^{2} \boldsymbol{\lambda}_{1}(\theta ; \omega) \\
\boldsymbol{f}_{r}^{\{b 0\}}(\theta ; \omega)=\theta \boldsymbol{\kappa}_{1}(\theta ; \omega)-\boldsymbol{\lambda}_{0}(\theta ; \omega)
\end{gathered}
$$




$$
\begin{aligned}
& \boldsymbol{f}_{\theta}^{\{a 2\}}(\theta ; \omega)=\boldsymbol{f}_{\theta}^{\{b 1\}}(\theta ; \omega)=\boldsymbol{f}_{\theta}^{\{c 0\}}(\theta ; \omega)=(1+\omega) \boldsymbol{\kappa}_{0}(\theta ; \omega) \\
& \boldsymbol{f}_{\theta}^{\{a \mid\}}(\theta ; \omega)=2 \boldsymbol{\kappa}_{0}(\theta ; \omega)+2(1+\omega) \theta \boldsymbol{\lambda}_{0}(\theta ; \omega) \\
& \boldsymbol{f}_{\theta}^{\{00\}}(\theta ; \omega)=2 \theta \lambda_{0}(\theta ; \omega)-(1+\omega) \theta^{2} \boldsymbol{\kappa}_{0}(\theta ; \omega) \\
& \boldsymbol{f}_{\theta}^{\{b 0\}}(\theta ; \omega)=\boldsymbol{\kappa}_{0}(\theta ; \omega)+(1+\omega) \theta \boldsymbol{\lambda}_{0}(\theta ; \omega) \\
& \boldsymbol{f}_{p}^{\{a 2\}}(\theta ; \omega)=\boldsymbol{f}_{p}^{\{b 1\}}(\theta ; \omega)=\boldsymbol{f}_{p}^{\{c 0\}}(\theta ; \omega)=(1-\omega)^{-1} \boldsymbol{\alpha}_{1}(\theta ; \omega) \\
& \boldsymbol{f}_{p}^{\{a 1\}}(\theta ; \omega)=\left[\frac{1}{1-\omega}\right] \boldsymbol{\alpha}_{2}(\theta ; \omega)+\left[\frac{2}{(1-\omega)^{2}}\right] \boldsymbol{\alpha}_{1}(\theta ; \omega)+4\left[\frac{1+\omega}{1-\omega}\right] \lambda_{1}(\theta ; \omega) \\
& \boldsymbol{f}_{p}^{\{a 0\}}(\theta ; \omega)=\left[\frac{1}{1-\omega}\right] \boldsymbol{\alpha}_{3}(\theta ; \omega)+\left[\frac{1}{(1-\omega)^{2}}\right] \boldsymbol{\alpha}_{2}(\theta ; \omega)+\left[\frac{2}{(1-\omega)^{3}}\right] \boldsymbol{\alpha}_{1}(\theta ; \omega) \\
& +4\left[\frac{1+\omega}{1-\omega}\right]\left[\lambda_{0}(\theta ; \omega)-\theta \kappa_{1}(\theta ; \omega)\right]+2\left[\frac{3+\omega}{(1-\omega)^{2}}\right] \lambda_{1}(\theta ; \omega) \\
& \boldsymbol{f}_{p}^{\{b 0\}}(\theta ; \omega)=\left[\frac{1}{1-\omega}\right] \boldsymbol{\alpha}_{4}(\theta ; \omega)+\left[\frac{1}{(1-\omega)^{2}}\right] \boldsymbol{\alpha}_{1}(\theta ; \omega)+2\left[\frac{1+\omega}{1-\omega}\right] \boldsymbol{\lambda}_{1}(\theta ; \omega) \\
& \boldsymbol{f}_{\tau}^{\{a 2\}}(\theta ; \omega)=\boldsymbol{f}_{\tau}^{\{b 1\}}(\theta ; \omega)=\boldsymbol{f}_{\tau}^{\{c 0\}}(\theta ; \omega)=\boldsymbol{\kappa}_{2}(\theta ; \omega)-\left(1-\omega^{2}\right) \boldsymbol{\kappa}_{0}(\theta ; \omega) \\
& \boldsymbol{f}_{\tau}^{\{a 1\}}(\theta ; \omega)=4 \omega \boldsymbol{\kappa}_{0}(\theta ; \omega)+4 \boldsymbol{\kappa}_{1}(\theta ; \omega)+2 \theta \boldsymbol{\lambda}_{2}(\theta ; \omega)-2\left(1-\omega^{2}\right) \theta \boldsymbol{\lambda}_{0}(\theta ; \omega) \text { (B 18) } \\
& \boldsymbol{f}_{\tau}^{\{a 0\}}(\theta ; \omega)=\left[4+\left(1-\omega^{2}\right) \theta^{2}\right] \kappa_{0}(\theta ; \omega)+4 \theta\left[\omega \lambda_{0}(\theta ; \omega)+\lambda_{1}(\theta ; \omega)\right] \\
& -\theta^{2} \boldsymbol{\kappa}_{2}(\theta ; \omega) \\
& \boldsymbol{f}_{\tau}^{\{b 0\}}(\theta ; \omega)=2 \boldsymbol{\kappa}_{1}(\theta ; \omega)+2 \omega \boldsymbol{\kappa}_{0}(\theta ; \omega)+\theta \boldsymbol{\lambda}_{2}(\theta ; \omega)-\left(1-\omega^{2}\right) \theta \boldsymbol{\lambda}_{0}(\theta ; \omega)
\end{aligned}
$$

and

$$
\begin{gathered}
\boldsymbol{\alpha}_{1}(\theta ; \omega)=(1+\omega)^{2} \lambda_{1}(\theta ; \omega)-\lambda_{3}(\theta ; \omega) \\
\boldsymbol{\alpha}_{2}(\theta ; \omega)=2(1+\omega)^{2}\left[\lambda_{0}(\theta ; \omega)-\theta \boldsymbol{\kappa}_{1}(\theta ; \omega)\right]-6 \boldsymbol{\lambda}_{2}(\theta ; \omega)+2 \theta \boldsymbol{\kappa}_{3}(\theta ; \omega) \\
\boldsymbol{\alpha}_{3}(\theta ; \omega)=6\left[\theta \boldsymbol{\kappa}_{2}(\theta ; \omega)-\boldsymbol{\lambda}_{1}(\theta ; \omega)\right]+\theta^{2} \boldsymbol{\lambda}_{3}(\theta ; \omega) \\
-(1+\omega)^{2} \theta\left[2 \boldsymbol{\kappa}_{0}(\theta ; \omega)+\theta \boldsymbol{\lambda}_{1}(\theta ; \omega)\right] \\
\boldsymbol{\alpha}_{4}(\theta ; \omega)=\theta \boldsymbol{\kappa}_{3}(\theta ; \omega)-3 \boldsymbol{\lambda}_{2}(\theta ; \omega)+(1+\omega)^{2}\left[\boldsymbol{\lambda}_{0}(\theta ; \omega)-\theta \boldsymbol{\kappa}_{1}(\theta ; \omega)\right] .
\end{gathered}
$$

The coefficient formulas (B 5)-(B 24) apply whenever $\omega \neq \pm 1$. For the special case $\omega=-1$ we have instead

$$
\begin{gathered}
\boldsymbol{f}_{r}^{\{a 2\}}(\theta ;-1)=\boldsymbol{f}_{r}^{\{b 1\}}(\theta ;-1)=\boldsymbol{f}_{r}^{\{c 0\}}(\theta ;-1)=2 \boldsymbol{\xi}(\theta)-\boldsymbol{\phi} \\
\boldsymbol{f}_{r}^{\{a 1\}}(\theta ;-1)=-4 \theta \boldsymbol{\eta}(\theta)-2 \boldsymbol{\xi}(\theta) \\
\boldsymbol{f}_{r}^{\{a 0\}}(\theta ;-1)=-2 \theta^{2} \boldsymbol{\xi}(\theta)+2 \theta \boldsymbol{\eta}(\theta)+2 \theta \boldsymbol{\zeta}(\theta)-\theta^{2} \boldsymbol{\phi} \\
\boldsymbol{f}_{r}^{\{b 0\}}(\theta ;-1)=-2 \theta \boldsymbol{\eta}(\theta)-\boldsymbol{\xi}(\theta) \\
\boldsymbol{f}_{\theta}^{\{a 2\}}(\theta ;-1)=\boldsymbol{f}_{\theta}^{\{b 1\}}(\theta ;-1)=\boldsymbol{f}_{\theta}^{\{c 0\}}(\theta ;-1)=0 \\
\boldsymbol{f}_{\theta}^{\{a 1\}}(\theta ;-1)=2 \boldsymbol{\eta}(\theta)+2 \zeta(\theta) \\
\boldsymbol{f}_{\theta}^{\{a 0\}}(\theta ;-1)=2 \theta \boldsymbol{\xi}(\theta) \\
\boldsymbol{f}_{\theta}^{\{b 0\}}(\theta ;-1)=\boldsymbol{\eta}(\theta)+\boldsymbol{\zeta}(\theta) \\
\boldsymbol{f}_{p}^{\{a 2\}}(\theta ;-1)=\boldsymbol{f}_{p}^{\{b\}}(\theta ;-1)=\boldsymbol{f}_{p}^{\{c 0\}}(\theta ;-1)=4 \boldsymbol{\xi}(\theta) \\
\boldsymbol{f}_{p}^{\{a 1\}}(\theta ;-1)=-8 \theta \boldsymbol{\eta}(\theta)-8 \boldsymbol{\xi}(\theta) \\
\boldsymbol{f}_{p}^{\{a 0\}}(\theta ;-1)=-4 \theta^{2} \boldsymbol{\xi}(\theta)+8 \theta \boldsymbol{\eta}(\theta) \\
\boldsymbol{f}_{p}^{\{b 0\}}(\theta ;-1)=-4 \theta \boldsymbol{\eta}(\theta)-4 \boldsymbol{\xi}(\theta)
\end{gathered}
$$




$$
\begin{gathered}
\boldsymbol{f}_{\tau}^{\{a 2\}}(\theta ;-1)=\boldsymbol{f}_{\tau}^{\{b 1\}}(\theta ;-1)=\boldsymbol{f}_{\tau}^{\{c 0\}}(\theta ;-1)=4 \boldsymbol{\eta}(\theta) \\
\boldsymbol{f}_{\tau}^{\{a 1\}}(\theta ;-1)=8 \theta \boldsymbol{\xi}(\theta)-12 \boldsymbol{\eta}(\theta)-4 \boldsymbol{\zeta}(\theta) \\
\boldsymbol{f}_{\tau}^{\{a 0\}}(\theta ;-1)=4\left(1-\theta^{2}\right) \boldsymbol{\eta}(\theta)-12 \theta \boldsymbol{\xi}(\theta)+4 \zeta(\theta) \\
\boldsymbol{f}_{\tau}^{\{b 0\}}(\theta ;-1)=4 \theta \boldsymbol{\xi}(\theta)-6 \boldsymbol{\eta}(\theta)-2 \zeta(\theta)
\end{gathered}
$$

in which we have used the following vector basis functions:

$$
\boldsymbol{\xi}(\theta)=\left(\begin{array}{c}
\sin (2 \theta) \\
-\cos (2 \theta) \\
0 \\
0
\end{array}\right), \quad \boldsymbol{\eta}(\theta)=\left(\begin{array}{c}
\cos (2 \theta) \\
\sin (2 \theta) \\
0 \\
0
\end{array}\right), \quad \zeta(\theta)=\left(\begin{array}{l}
0 \\
0 \\
1 \\
\theta
\end{array}\right), \quad \boldsymbol{\phi}=\left(\begin{array}{l}
0 \\
0 \\
0 \\
1
\end{array}\right)
$$

The case $\omega=1$ is unusual for two reasons. First, the pressure field does not fit within the general form of (B 3),

$$
\begin{aligned}
p(r, \theta ; 1, \boldsymbol{a}, \boldsymbol{b}, \boldsymbol{c})= & {\left[\boldsymbol{a} \cdot \boldsymbol{f}_{p}^{\{a 2\}}(\theta ; 1)\right](\ln r)^{3}+\left[\boldsymbol{a} \cdot \boldsymbol{f}_{p}^{\{a 1\}}(\theta ; 1)+\boldsymbol{b} \cdot \boldsymbol{f}_{p}^{\{b 1\}}(\theta ; 1)\right](\ln r)^{2} } \\
& +\left[\boldsymbol{a} \cdot \boldsymbol{f}_{p}^{\{a 0\}}(\theta ; 1)+\boldsymbol{b} \cdot \boldsymbol{f}_{p}^{\{b 0\}}(\theta ; 1)+\boldsymbol{c} \cdot \boldsymbol{f}_{p}^{\{c 0\}}(\theta ; 1)\right] \ln r,
\end{aligned}
$$

although $v_{r}, v_{\theta}$ and $\tau_{\theta r}$ are given by (B 1), (B 2) and (B 4) with $\omega=1$. Second, there are only 10 free constants instead of 12 , whereby the third and fourth components of the vector coefficient functions $\boldsymbol{f}_{r}^{\{b 1\}}(\theta ; 1), \boldsymbol{f}_{r}^{\{b 0\}}(\theta ; 1), \boldsymbol{f}_{\theta}^{\{b 1\}}(\theta ; 1), \boldsymbol{f}_{\theta}^{\{b 0\}}(\theta ; 1), \boldsymbol{f}_{p}^{\{b 1\}}(\theta ; 1)$, $\boldsymbol{f}_{p}^{\{b 0\}}(\theta ; 1), \boldsymbol{f}_{\tau}^{\{b 1\}}(\theta ; 1), \boldsymbol{f}_{\tau}^{\{b 0\}}(\theta ; 1)$ all vanish. This renders the constants $b_{3}$ and $b_{4}$ in (3.14) superfluous:

$$
\begin{aligned}
& \boldsymbol{f}_{r}^{\{a 2\}}(\theta ; 1)=\boldsymbol{f}_{r}^{\{c 0\}}(\theta ; 1)=\boldsymbol{f}_{r}^{\{a 2\}}(\theta ;-1) \\
& \boldsymbol{f}_{r}^{\{a 1\}}(\theta ; 1)=2 \boldsymbol{\phi}-\boldsymbol{f}_{r}^{\{a 1\}}(\theta ;-1) \\
& \boldsymbol{f}_{r}^{\{a 0\}}(\theta ; 1)=\boldsymbol{f}_{r}^{\{a 0\}}(\theta ;-1) \\
& \boldsymbol{f}_{r}^{\{b 1\}}(\theta ; 1)=2 \xi(\theta) \\
& \boldsymbol{f}_{r}^{\{b 0\}}(\theta ; 1)=-\boldsymbol{f}_{r}^{\{b 0\}}(\theta ;-1) \\
& \boldsymbol{f}_{\theta}^{\{a 2\}}(\theta ; 1)=\boldsymbol{f}_{\theta}^{\{c 0\}}(\theta ; 1)=\boldsymbol{f}_{\theta}^{\{a 1\}}(\theta ;-1) \\
& \boldsymbol{f}_{\theta}^{\{a 1\}}(\theta ; 1)=-4 \theta \boldsymbol{\xi}(\theta)+2 \eta(\theta)-2 \boldsymbol{\zeta}(\theta) \\
& \boldsymbol{f}_{\theta}^{\{a 0\}}(\theta ; 1)=-2 \theta^{2} \boldsymbol{\eta}(\theta)-2 \theta \boldsymbol{\xi}(\theta)-2\left(1+\theta^{2}\right) \boldsymbol{\zeta}(\theta)+\frac{4}{3} \theta^{3} \boldsymbol{\phi} \\
& \boldsymbol{f}_{\theta}^{\{b 1\}}(\theta ; 1)=2 \boldsymbol{\eta}(\theta) \\
& \boldsymbol{f}_{\theta}^{\{b 0\}}(\theta ; 1)=-2 \theta \boldsymbol{\xi}(\theta)+\boldsymbol{\eta}(\theta) \\
& \boldsymbol{f}_{p}^{\{a 2\}}(\theta ; 1)=-\frac{4}{3} \boldsymbol{\phi} \\
& \boldsymbol{f}_{p}^{\{a 0\}}(\theta ; 1)=8 \theta \zeta(\theta)+\left(8-4 \theta^{2}\right) \boldsymbol{\phi} \\
& \boldsymbol{f}_{p}^{\{c 0\}}(\theta ; 1)=-4 \phi \\
& \boldsymbol{f}_{p}^{\{a 1\}}(\theta ; 1)=\boldsymbol{f}_{p}^{\{b 1\}}(\theta ; 1)=\boldsymbol{f}_{p}^{\{b 0\}}(\theta ; 1)=\boldsymbol{f}_{p}^{\{c 0\}}(\theta ; 1)=0 \\
& \boldsymbol{f}_{\tau}^{\{a 2\}}(\theta ; 1)=\boldsymbol{f}_{\tau}^{\{b 1\}}(\theta ; 1)=\boldsymbol{f}_{\tau}^{\{c 0\}}(\theta ; 1)=\boldsymbol{f}_{\tau}^{\{a 2\}}(\theta ;-1) \\
& \boldsymbol{f}_{\tau}^{\{a 1\}}(\theta ; 1)=-\boldsymbol{f}_{\tau}^{\{a 1\}}(\theta ;-1) \\
& \boldsymbol{f}_{\tau}^{\{a 0\}}(\theta ; 1)=\boldsymbol{f}_{\tau}^{\{a 0\}}(\theta ;-1)-4 \zeta(\theta) \\
& \boldsymbol{f}_{\tau}^{\{b 0\}}(\theta ; 1)=-\boldsymbol{f}_{\tau}^{\{b 0\}}(\theta ;-1)-2 \zeta(\theta) \text {. }
\end{aligned}
$$




\section{Appendix C. Building-block solutions from $§ 4.1$ in Cartesian coordinates}

We have

$$
\begin{aligned}
& p^{\{1\}}(x, y)=1-\left(\frac{2}{\pi}\right) \frac{x}{r^{2}} \\
& v_{x}^{\{1\}}(x, y)=\frac{y^{2}}{\pi r^{2}} \\
& v_{y}^{\{1\}}(x, y)=\frac{\theta}{\pi}-1-\frac{x y}{\pi r^{2}} \\
& p^{\{2\}}(x, y)=\frac{\ln r}{r^{4}}\left[\frac{2 y^{2}-2 x^{2}}{\pi}\right]+\frac{1}{r^{4}}\left[\frac{2 x^{2}-4 x y \theta-2 y^{2}}{\pi}\right] \\
& v_{x}^{\{2\}}(x, y)=\frac{\ln r}{r^{4}}\left[\frac{2 x y^{2}}{\pi}\right]+\frac{1}{r^{4}}\left[-x^{2} y\left(1+\frac{\theta}{\pi}\right)-\frac{2 x y^{2}}{\pi}-y^{3}\left(1-\frac{\theta}{\pi}\right)\right] \\
& v_{y}^{\{2\}}(x, y)=\frac{\ln r}{r^{4}}\left[\frac{2 y^{3}}{\pi}\right]+\frac{1}{r^{4}}\left[x^{3}\left(1-\frac{\theta}{\pi}\right)+\frac{x^{2} y}{\pi}+x y^{2}\left(1-\frac{3 \theta}{\pi}\right)-\frac{y^{3}}{\pi}\right] \\
& p^{\{3\}}(x, y)=\frac{2 x^{2}-2 y^{2}}{\pi r^{4}} \\
& v_{x}^{\{3\}}(x, y)=-\frac{2 x y^{2}}{\pi r^{4}} \\
& v_{y}^{\{3\}}(x, y)=-\frac{2 y^{3}}{\pi r^{4}} \\
& p^{\{4\}}(x, y)=-\frac{8 x y}{\pi r^{4}} \\
& v_{x}^{\{4\}}(x, y)=-\frac{4 x^{2} y}{\pi r^{4}} \\
& v_{y}^{\{4\}}(x, y)=-\frac{4 x y^{2}}{\pi r^{4}} \\
& p^{\{5\}}(x, y)=\frac{\ln r}{r^{6}}\left[\frac{4 x}{\pi}\left(3 y^{2}-x^{2}\right)\right]+\frac{1}{r^{6}}\left[\frac{5 x}{3 \pi}\left(3 y^{2}-x^{2}\right)+\frac{4 y \theta}{\pi}\left(y^{2}-3 x^{2}\right)\right] \\
& v_{x}^{\{5\}}(x, y)=\frac{\ln r}{r^{6}}\left[\frac{2 y^{2}}{\pi}\left(3 x^{2}-y^{2}\right)\right]+\frac{1}{r^{6}}\left[-2 x^{3} y\left(1+\frac{\theta}{\pi}\right)+\frac{5 x^{2} y^{2}}{2 \pi}\right. \\
& \left.-2 x y^{3}\left(1-\frac{3 \theta}{\pi}\right)-\frac{5 y^{4}}{6 \pi}\right] \\
& v_{y}^{\{5\}}(x, y)=\frac{\ln r}{r^{6}}\left[\frac{8 x y^{3}}{\pi}\right]+\frac{1}{r^{6}}\left[x^{4}\left(1-\frac{\theta}{\pi}\right)+\frac{x^{3} y}{\pi}-\frac{6 x^{2} y^{2} \theta}{\pi}\right. \\
& \left.+\frac{13 x y^{3}}{3 \pi}-y^{4}\left(1-\frac{3 \theta}{\pi}\right)\right]
\end{aligned}
$$




$$
\begin{aligned}
& p^{\{6\}}(x, y)=\frac{(\ln r)^{2}}{r^{6}}\left[\frac{x}{\pi}\left(6 y^{2}-2 x^{2}\right)\right] \\
& +\frac{\ln r}{r^{6}}\left[y\left(12 x^{2}-4 y^{2}\right)\left(1-\frac{\theta}{\pi}\right)+\frac{x}{\pi}\left(2 x^{2}-6 y^{2}\right)\right] \\
& +\frac{1}{r^{6}}\left[x^{3}\left(\frac{1}{\pi}-4 \theta+\frac{2 \theta^{2}}{\pi}\right)+x^{2} y\left(5+\frac{6 \theta}{\pi}\right)\right. \\
& \left.-x y^{2}\left(\frac{3}{\pi}-12 \theta+\frac{6 \theta^{2}}{\pi}\right)-y^{3}\left(\frac{5}{3}+\frac{2 \theta}{\pi}\right)\right] \\
& v_{x}^{\{6\}}(x, y)=\frac{(\ln r)^{2}}{r^{6}}\left[\frac{y^{2}\left(3 x^{2}-y^{2}\right)}{\pi}\right] \\
& +\frac{\ln r}{r^{6}}\left[x y\left(2 x^{2}-6 y^{2}\right)\left(1-\frac{\theta}{\pi}\right)+\frac{y^{2}}{\pi}\left(y^{2}-3 x^{2}\right)\right] \\
& +\frac{1}{r^{6}}\left[x^{3} y\left(\frac{8}{3}+\frac{\theta}{\pi}\right)-x^{2} y^{2}\left(\frac{3}{2 \pi}-6 \theta+\frac{3 \theta^{2}}{\pi}\right)\right. \\
& \left.-x y^{3}\left(\frac{2}{3}+\frac{3 \theta}{\pi}\right)+y^{4}\left(\frac{1}{2 \pi}-2 \theta+\frac{\theta^{2}}{\pi}\right)\right] \\
& v_{y}^{\{6\}}(x, y)=\frac{(\ln r)^{2}}{r^{6}}\left[\frac{4 x y^{3}}{\pi}\right] \\
& +\frac{\ln r}{r^{6}}\left[\left(x^{4}+6 x^{2} y^{2}-3 y^{4}\right)\left(1-\frac{\theta}{\pi}\right)+\frac{x y}{\pi}\left(x^{2}-3 y^{2}\right)\right] \\
& +\frac{1}{r^{6}}\left[x^{2} y^{2}\left(\frac{5}{2}+\frac{3 \theta}{\pi}\right)-x y^{3}\left(\frac{2}{\pi}-8 \theta+\frac{4 \theta^{2}}{\pi}\right)\right. \\
& \left.-y^{4}\left(\frac{5}{6}+\frac{\theta}{\pi}\right)\right] \\
& p^{\{7\}}(x, y)=\frac{1}{r^{6}}\left[y\left(6 x^{2}-2 y^{2}\right)\right] \\
& v_{x}^{\{7\}}(x, y)=\frac{1}{r^{6}}\left[2 x y\left(x^{2}-y^{2}\right)\right] \\
& v_{y}^{\{7\}}(x, y)=\frac{1}{r^{6}}\left[y^{2}\left(3 x^{2}-y^{2}\right)\right] \\
& p^{\{8\}}(x, y)=\frac{1}{r^{6}}\left[x\left(2 x^{2}-6 y^{2}\right)\right] \\
& v_{x}^{\{8\}}(x, y)=\frac{1}{r^{6}}\left[y^{2}\left(y^{2}-3 x^{2}\right)\right] \\
& v_{y}^{\{8\}}(x, y)=\frac{1}{r^{6}}\left[-4 x y^{3}\right] \text {. }
\end{aligned}
$$

\section{Appendix D. Sample program that uses the Fortran 2003 library listed in the supplementary material}

Here we illustrate the calling sequences for the relevant subroutines with a short sample program: 


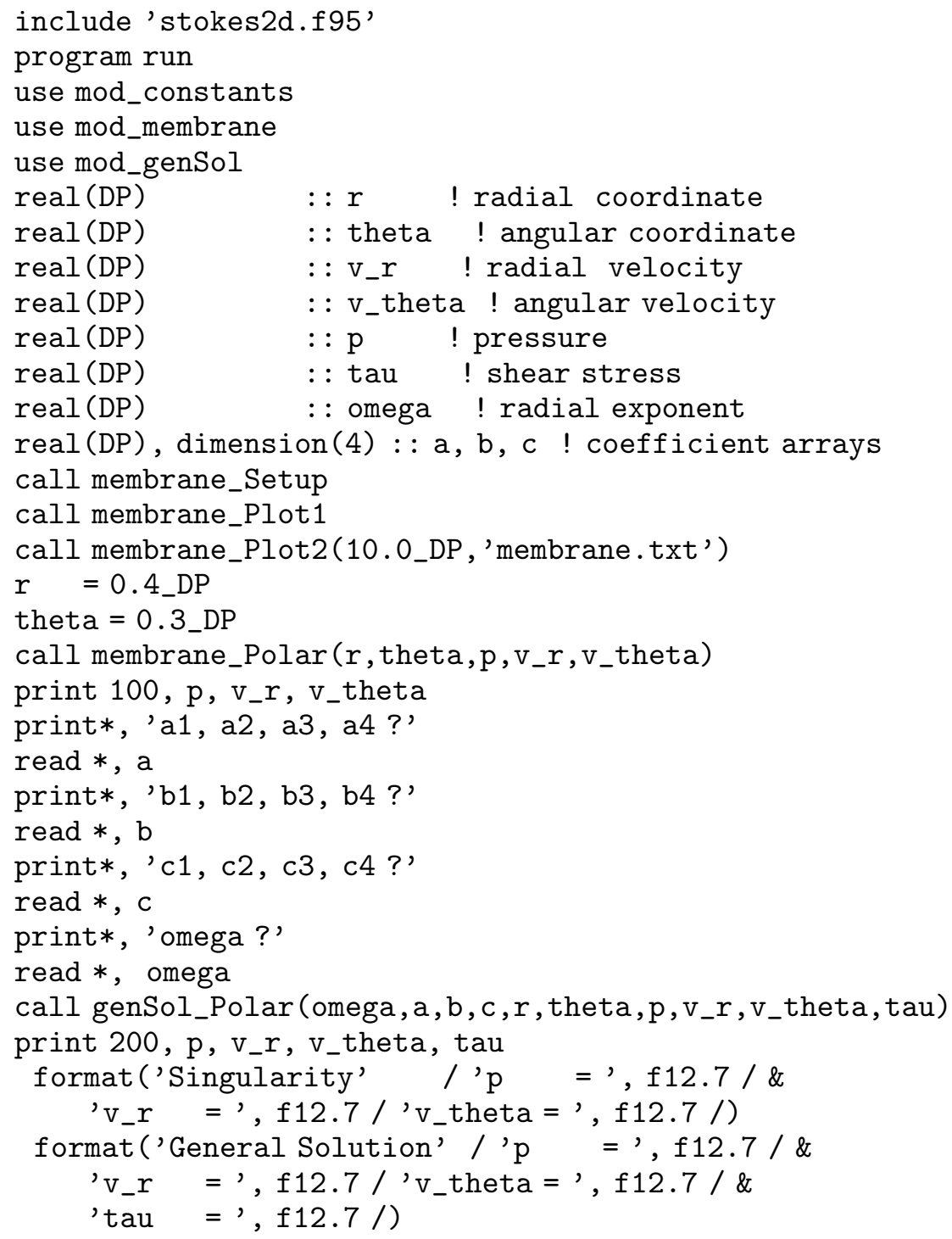

end program run

\section{REFERENCES}

AleKsidze, M. A. 1966 On approximate solutions of a certain mixed boundary value problem in the theory of harmonic functions. Differ. Equ. 2, 515-518.

Alves, C. J. S. 2009 On the choice of source points in the method of fundamental solutions. Engng Anal. Bound. Elem. 33, 1348-1361.

Alves, C. J. S. \& Silvestre, A. L. 2004 Density results using Stokeslets and a method of fundamental solutions for the Stokes equations. Engng Anal. Bound. Elem. 28, 1245-1252.

Asghar, S., Mushtaq, M. \& Hayat, T. 2010 Flow in a slowly deforming channel with weak permeability: an analytical approach. Nonlinear Anal. Real World Applics 11, 555-561.

Beavers, G. S. \& Joseph, D. D. 1967 Boundary conditions at a naturally permeable wall. J. Fluid Mech. 30, 197-207.

Beavers, G. S., Sparrow, E. M. \& Masha, B. A. 1974 Boundary condition at a porous surface which bounds a fluid flow. AIChE J. 20, 596-597. 
Berman, A. S. 1953 Laminar flow in channels with porous walls. J. Appl. Phys. 24, 1232-1235.

Betyaev, S. K. 2002 Fluid flow within permeable boundaries. Fluid Dyn. 37, 695-704.

Bogomolny, A. 1985 Fundamental solutions method for elliptic boundary value problems. SIAM J. Numer. Anal. 22, 644-669.

Borsi, I., FARINA, A. \& FASANO, A. 2011 Incompressible laminar flow through hollow fibers: a general study by means of a two-scale approach. Z. Angew. Math. Phys. 62, 681-706.

Brady, J. F. 1984 Flow development in a porous channel and tube. Phys. Fluids 27, 1061-1067.

BRUNS, F. W. 1979 Numerical calculation on creeping flow in a closed cylindrical cavity. Comput. Fluids 7, 257-265.

Bujurke, N. M., Madalli, V. S. \& Mulimani, B. G. 1998 Long series analysis of laminar flow through parallel and uniformly porous walls of different permeability. Comput. Meth. Appl. Mech. Engng 160, 39-56.

Burgess, G. \& Mahajerin, E. 1984 Rotational fluid flow using a least squares collocation technique. Comput. Fluids 4, 311-317.

Chellam, S. \& LiU, M. 2006 Effect of slip on existence, uniqueness, and behavior of similarity solutions for steady incompressible laminar flow in porous tubes and channels. Phys. Fluids 18, 083601.

Chellam, S., Wiesner, M. R. \& Dawson, C. 1992 Slip at a uniformly porous boundary: effect on fluid flow and mass transfer. J. Engng Maths 26, 481-492.

Chellam, S., Wiesner, M. R. \& Dawson, C. 1995 Laminar flow in porous ducts. Rev. Chem. Engng 11, 53-99.

Chen, N., Gunzburger, M. \& Wang, X. 2010 Asymptotic analysis of the differences between the Stokes-Darcy system with different interface conditions and the Stokes-Brinkman system. J. Math. Anal. Applics 368, 658-676.

Damak, K, Ayadi, A, Zeghmati, B \& Schmitz, P 2004a Concentration polarisation in tubular membranes - a numerical approach. Desalination 171, 139-153.

Damak, K, Ayadi, A, Zeghmati, B \& Schmitz, P 2004b A new Navier-Stokes and Darcy's law combined model for fluid flow in crossflow filtration tubular membranes. Desalination 161, $67-77$.

Dassios, G. \& Hadjinicolaou, M. 2002 Multipole expansions in Stokes flow. Intl J. Engng Sci. 40, 223-229.

Dauenhauer, E. C. \& Majdalani, J. 2003 Exact self-similarity solution of the Navier-Stokes equations for a porous channel with orthogonally moving walls. Phys. Fluids 15, $1485-1495$.

DA̧BROŚ, T. 1985 A singularity method for calculating hydrodynamic forces and particle velocities in low-Reynolds-number flows. J. Fluid Mech. 156, 1-21.

DEMPSEY, J. P. 1995 Power-logarithmic stress singularities at bi-material corners and interface cracks. J. Adhes. Sci. Technol. 9, 253-265.

Dempsey, J. P. \& Sinclair, G. B. 1979 On the stress singularities in the plane elasticity of the composite wedge. J. Elast. 9, 373-391.

Dempsey, J. P. \& SinclaiR, G. B. 1981 On the singular behaviour at the vertex of a bi-material wedge. J. Elast. 11, 317-327.

Deng, C. \& MARTinez, D. M. 2005 Viscous flow in a channel partially filled with a porous medium and with wall suction. Chem. Engng Sci. 60, 329-336.

FAirweather, G. \& Karageorghis, A. 1998 The method of fundamental solutions for elliptic boundary value problems. Adv. Comput. Maths 9, 69-95.

Ferro, S. \& GNAVI, G. 2000 Spatial stability of similarity solutions for viscous flows in channels with porous walls. Phys. Fluids 12, 797-802.

FloRYAN, J. M. \& CZECHOWSKI, L. 1995 On the numerical treatment of corner singularity in the vorticity field. J. Comput. Phys. 118, 222-228.

Galowin, L. S., Fletcher, L. S. \& DeSantis, M. J. 1974 Investigation of laminar flow in a porous pipe with variable wall suction. AIAA J. 12, 1585-1589.

Geraldes, V., Semiño, V. \& DE Pinho, M. N. 2002a The effect of the ladder-type spacers configuration in NF spiral-wound modules on the concentration boundary layers disruption. Desalination 146, 187-194. 
Geraldes, V., Semiao, V. \& De Pinho, M. N. $2002 b$ The effect on mass transfer of momentum and concentration boundary layers at the entrance region of a slit with a nanofiltration membrane wall. Chem. Engng Sci. 57, 735-748.

Geraldes, V., Semiño, V. \& DE Pinho, M. N. 2002c Flow management in nanofiltration spiral wound modules with ladder-type spacers. J. Membr. Sci. 203, 87-102.

Gluckman, M. J., Pfeffer, R. \& Weinbaum, S. 1971 A new technique for treating multiparticle slow viscous flow: axisymmetric flow past spheres and spheroids. J. Fluid Mech. 50, 705-740.

GuptA, B. K. \& LeVy, E. K. 1976 Symmetrical laminar channel flow with wall suction. Trans. ASME: J. Fluids Engng 98, 469-474.

Gupta, M. M., Manohar, R. P. \& Noble, B. 1981 Nature of viscous flows near sharp corners. Comput. Fluids 9, 379-388.

Gupte, S. K. \& ADVANI, S. G. 1997 Flow near the permeable boundary of a porous medium: an experimental investigation using LDA. Exp. Fluids 22, 408-422.

HALDENWANG, P. 2007 Laminar flow in a two-dimensional plane channel with local pressuredependent crossflow. J. Fluid Mech. 593, 463-473.

Hamza, E. A. 1999 Suction and injection effects on a similar flow between parallel plates. J. Phys. D: Appl. Phys. 32, 656-663.

HAN, P. S. \& Olson, M. D. 1987 An adaptive boundary element method. Intl J. Numer. Meth. Engng 24, 1187-1202.

Hancock, C., Lewis, E. \& Moffatt, H. K. 1981 Effects of inertia in forced corner flows. J. Fluid Mech. 112, 315-327.

HAPpel, J. \& BRenneR, H. 1991 Low Reynolds Number Hydrodynamics: With Special Applications to Particulate Media. Kluwer.

HARPER, J. F. \& WAKE, G. C. 1983 Stokes flow between parallel plates due to a transversely moving end wall. IMA J. Appl. Maths 30, 141-149.

HAWA, T. \& RUSAK, Z. 2002 Numerical-asymptotic expansion matching for computing a viscous flow around a sharp expansion corner. Theor. Comput. Fluid Dyn. 15, 265-281.

Hills, C. P. \& Moffatt, H. K. 2000 Rotary honing: a variant of the Taylor paint-scraper problem. J. Fluid Mech. 418, 119-135.

Hwang, T.-W. \& WANG, C.-A. 1992 On multiple solutions for Berman's problem. Proc. R. Soc. Edin. 121A, 219-230.

Ingham, D. B. \& Kelmanson, M. A. 1984 Boundary Integral Equation Analyses of Singular, Potential, and Biharmonic Problems, Lecture Notes in Engineering, vol. 7. Springer.

Jones, I. P. 1973 Low Reynolds number flow past a porous spherical shell. Math. Proc. Camb. Phil. Soc. 73, 231-238.

KIM, A. S. 2007 Permeate flux inflection due to concentration polarization in crossflow membrane filtration: a novel analytic approach. Eur. Phys. J. E 24, 331-341.

Kim, S. \& Karrila, S. J. 2005 Microhydrodynamics: Principles and Selected Applications. Dover.

KING, J. R. \& CoX, S. M. 2005 Self-similar 'stagnation point' boundary layer flows with suction or injection. Stud. Appl. Maths 115, 73-107.

KoŁodziej, J. A. \& KLeIBer, M. 1989 Boundary collocation method vs FEM for some harmonic 2-d problems. Comput. Struct. 33, 155-168.

Kostoglou, M. \& Karabelas, A. J. 2008 On the structure of the single-phase flow field in hollow fiber membrane modules during filtration. J. Membr. Sci. 322, 128-138.

KUPRADZE, V. D. 1964 A method for the approximate solution of limiting problems in mathematical physics. USSR Comput. Maths Math. Phys. 4, 199-205.

Kupradze, V. D. \& AleKsidze, M. A. 1964 The method of functional equations for the approximate solution of certain boundary value problems. USSR Comput. Maths Math. Phys. 4, 82-126.

LARson, R. E. \& Higdon, J. J. L. 1986 Microscopic flow near the surface of two-dimensional porous media. Part 1. Axial flow. J. Fluid Mech. 166, 449-472.

LARson, R. E. \& Higdon, J. J. L. 1987 Microscopic flow near the surface of two-dimensional porous media. Part 2. Transverse flow. J. Fluid Mech. 178, 119-136.

LI, Z. L. \& WANG, CH. 2010 Particular solutions of a two-dimensional infinite wedge for various boundary conditions with weak singularity. Trans. ASME: J. Appl. Mech. 77, 011004. 
LiU, Q. \& Prosperetti, A. 2011 Pressure-driven flow in a channel with porous walls. J. Fluid Mech. 679, 77-100.

LU, C. 1997 On the asymptotic solution of laminar channel flow with large suction. SIAM J. Math. Anal. 28, 1113-1134.

Majdalani, J., Zhou, C. \& Dawson, C. A. 2002 Two-dimensional viscous flow between slowly expanding or contracting walls with weak permeability. J. Biomech. 35, 1399-1403.

Mathon, R. \& Johnston, R. L. 1977 The approximate solution of elliptic boundary-value problems by fundamental solutions. SIAM J. Numer. Anal. 14, 638-650.

Mellis, R., Gill, W. N. \& Belfort, G. 1993 Fluid dynamics in a tubular membrane: theory and experiment. Chem. Engng Commun. 122, 103-125.

Miranda, J. M. \& Campos, J. B. L. M. 2001 An improved numerical scheme to study mass transfer over a separation membrane. J. Membr. Sci. 188, 49-59.

Moffatt, H. K. $1964 a$ Viscous and resistive eddies near a sharp corner. J. Fluid Mech. 18, 1-18.

Moffatt, H. K. $1964 b$ Viscous eddies near a sharp corner. Arch. Mech. Stosowanej 2, 365-372.

Moffatt, H. K. \& DufFy, B. R. 1980 Local similarity solutions and their limitations. J. Fluid Mech. 96, 299-313.

NeAle, G. \& NADER, W. 1974 Practical significance of Brinkman's extension of Darcy's law: coupled parallel flows within a channel and a bounding porous medium. Can. J. Chem. Engng 52, 475-478.

NIELD, D. A. 1983 The boundary correction for the Rayleigh-Darcy problem: limitations of the Brinkman equation. J. Fluid Mech. 128, 37-46.

NiELD, D. A. 2009 The Beavers-Joseph boundary condition and related matters: a historical and critical note. Transp. Porous Med. 78, 537-540.

Nitsche, L. C. \& Brenner, H. 1990 Hydrodynamics of particulate motion in sinusoidal pores via a singularity method. AIChE J. 36, 1403-1419.

Nitsche, L. C. \& Zhuge, S. 1995 Hydrodynamics and selectivity of antipolarization dialysis. Chem. Engng Sci. 50, 2731-2746.

Pak, A., Mohammadi, T., Hosseinalipour, S. M. \& Allahdini, V. 2008 CFD modeling of porous membranes. Desalination 222, 482-488.

PAN, F. \& ACRivos, A. 1967 Steady flows in rectangular cavities. J. Fluid Mech. 28, $643-655$.

PARTHASARATHI, P. 2008 Asymptotic and particle methods in nonlinear transport phenomena: membrane separations and drop dynamics. PhD thesis, The University of Illinois at Chicago.

Patil, S. K. R. \& NG, T. T. 2010 Control of separation using spanwise periodic porosity. AIAA J. 48, 174-187.

De Pinho, M. N., Semião, V. \& Geraldes, V. 2002 Integrated modelling of transport processes in fluid/nanofiltration membrane systems. J. Membr. Sci. 206, 189-200.

PozRIKIDIS, C. 1992 Boundary Integral and Singularity Methods for Linearized Viscous Flow. Cambridge University Press.

PozRIKIDIS, C. 2001 Shear flow over a particulate or fibrous plate. J. Engng Maths 39, 3-24.

POZRIKIDIS, C. 2005 Effect of membrane thickness on the slip and drift velocity in parallel shear flow. J. Fluids Struct. 20, 177-187.

PozRikidis, C. 2010 Stokes flow through a permeable tube. Arch. Appl. Mech. 80, 323-333.

Raghunathan, S. \& CoOper, R. K. 2000 Passive boundary layer control with slots in short diffusers. Trans. ASME: J. Fluids Engng 122, 177-179.

Raghunathan, S., McIllain, S. T. \& Mabey, D. G. 1991 Wide angle diffusers with passive boundary-layer control. Aeronaut. J. 95, 28-34.

Robinson, W. A. 1976 The existence of multiple solutions for the laminar flow in a uniformly porous channel with suction at both walls. J. Engng Maths 10, 23-40.

SAAVEDRA, I. \& POWER, H. 2003 Multipole fast algorithm for the least-squares approach of the method of fundamental solutions for three-dimensional harmonic problems. Numer. Meth. Partial Differ. Equ. 19, 828-845.

SAAVEDRA, I. \& Power, H. 2004 Adaptive refinement scheme for the least-squares approach of the method of fundamental solution for three-dimensional harmonic problems. Engng Anal. Bound. Elem. 28, 1123-1133. 
SAFFMAN, P. G. 1971 On the boundary condition at the surface of a porous medium. Stud. Appl. Maths 50, 93-101.

Sahraoui, M. \& KaVIANY, M. 1992 Slip and no-slip velocity boundary conditions at interface of porous, plain media. Intl J. Heat Mass Transfer 35, 927-943.

SCHMITZ, P. \& PRAT, M. 1995 3-d laminar stationary flow over a porous surface with suction: description at pore level. AIChE J. 41, 2212-2226.

ShapeEV, A. V. \& LiN, P. 2009 An asymptotic fitting finite element method with exponential mesh refinement for accurate computation of corner eddies in viscous flows. SIAM J. Sci. Comput. 31, 1874-1900.

Sherwood, T. K., Brian, P. L. T., Fisher, R. E. \& Dresner, L. 1965 Salt concentration at phase boundaries in desalination by reverse osmosis. I\&EC Fundam. 4, 113-118.

ShI, J.-M., BREUER, M. \& DURST, F. 2004 A combined analytical-numerical method for treating corner singularities in viscous flow predictions. Intl J. Numer. Meth. Fluids 45, 659-688.

SHIH, K.-G. 1987 On the existence of solutions of an equation arising in the theory of laminar flow in a uniformly porous channel with injection. SIAM J. Appl. Maths 47, 526-533.

SINClAIR, G. B. 2010 On the pressure and stress singularities induced by steady flows of a pair of nonmiscible, incompressible, viscous fluids in contact with a wall. Acta Mech. Sin. 26, 669-673.

Sinclair, G. B., ChI, X. \& SHIH, T. I.-P. 2009 On the pressure and stress singularities induced by steady flows of incompressible viscous fluids. Acta Mech. Sin. 25, 451-462.

SingH, R. \& LAURENCE, R. L. $1979 a$ Influence of slip velocity at a membrane surface on ultrafiltration performance - I. Channel flow system. Intl J. Heat Mass Transfer 22, 721-729.

SINGH, R. \& LAURENCE, R. L. $1979 b$ Influence of slip velocity at a membrane surface on ultrafiltration performance - II. Tube flow system. Intl J. Heat Mass Transfer 22, 731-737.

SKALAK, F. M. \& WANG, C.-Y. 1978 On the nonunique solutions of laminar flow through a porous tube or channel. SIAM J. Appl. Maths 34, 535-544.

Stampouloglou, I. H. \& Theotokoglou, E. E. 2010 Additional separated-variable solutions of the biharmonic equation in polar coordinates. Trans ASME: J. Appl. Mech. 77, 021003.

TAYLOR, G. 1956 Fluid flow in regions bounded by porous surfaces. Proc. R. Soc. Lond. A. 234, 456-475.

TAYLOR, G. I. 1960 Similarity solutions of hydrodynamic problems. In Aeronaut. Astronaut. (Durand Anniv. Vol.), pp. 21-28. Pergmon.

TAYLOR, G. I. 1962 On scraping viscous fluid from a plane surface. Reprinted in The Scientic Papers of Sir Geoffrey Ingram Taylor, vol. IV (ed. G. K. Batchelor), pp. 410-413. Cambridge University Press.

Taylor, C. L., Banks, W. H. H., Zaturska, M. B. \& Drazin, P. G. 1991 Three-dimensional flow in a porous channel. Q. J. Mech. Appl. Maths 44, 105-133.

TERriLl, R. M. 1964 Laminar flow in a uniformly porous channel. Aeronaut. Q. 15, 299-310.

WeIssberg, H. L. 1959 Laminar flow in the entrance region of a porous pipe. Phys. Fluids $\mathbf{2}$, $510-516$.

Yan, Z.-Y., Weinbaum, S., Ganatos, P. \& Pfeffer, R. 1987 The three-dimensional hydrodynamic interaction of a finite sphere with a circular orifice at low Reynolds number. J. Fluid Mech. 174, 39-68.

YeH, H. M. \& ChEnG, T. W. 1999 Analysis of the slip effect on the permeate flux in membrane ultrafiltration. J. Membr. Sci. 154, 41-51.

YuAn, S. W. \& Finkelstein, A. B. 1956 Laminar pipe flow with injection and suction through a porous wall. Trans. ASME 78, 719-724.

YuCEL, N. \& TURKoglu, H. 1995 Numerical analysis of fluid flow and mass transfer in a channel with a porous bottom wall. Intl J. Numer. Meth. Fluids 21, 391-399.

ZaturskA, M. B. \& BANKs, W. H. H. 1995 Suction-driven flow in a porous pipe. Z. Angew. Math. Mech. 75, 21-30.

Zaturska, M. B., Drazin, P. G. \& BAnks, W. H. H. 1988 On the flow of a viscous fluid driven along a channel by suction at porous walls. Fluid Dyn. Res. 4, 151-178.

Zheng, L., ZhaO, N. \& ZHANG, X. 2007 Asymptotic solutions for laminar flow in a channel with uniformly accelerating rigid porous walls. J. Univ. Sci. Technol. Beijing 14, 405-409. 